(C) American Psychological Association, 2020. This paper is not the copy of record and may not exactly replicate the authoritative document published in the APA journal. Please do not copy or cite without author's permission. The final article is available, upon publication, at: http://dx.doi.org/10.1037/xge0000971

5

\title{
Memory suppression and its deficiency in psychological disorders: A focused meta-analysis
}

\author{
Davide F. Stramaccia ${ }^{a}$, Ann-Kristin Meyer ${ }^{a}$, Katharina M. Rischer ${ }^{b}$, \\ 10 Jonathan M. Fawcett ${ }^{c}$, and Roland G. Benoit ${ }^{a}$
}

${ }^{a}$ Max Planck Research Group: Adaptive Memory, Max Planck Institute for Human Cognitive and Brain Sciences, 04103 Leipzig, Germany.

bepartment of Behavioural and Cognitive Sciences, Université du Luxembourg Maison des Sciences

Humaines 11, Porte des Sciences L-4366 Esch-sur-Alzette, Luxembourg.

${ }^{c}$ Department of Psychology, Memorial University of Newfoundland, 230 Elizabeth Ave, St. John's, NL A1C 5S7, Canada.

\section{Contacts}

$\begin{array}{ll}\text { Davide F. Stramaccia: } & \text { stramaccia@cbs.mpg.de } \\ \text { Roland G. Benoit: } & \text { rbenoit@cbs.mpg.de }\end{array}$

Author Note: All data, $R$ analysis scripts, and tables are available at the Open Science Framework (https://osf.io/f89ur/). 


\section{Abstract}

It is still debated whether suppressing the retrieval of unwanted memories causes forgetting and whether this constitutes a beneficial mechanism. To shed light on these two questions, we scrutinize the evidence for such suppression-induced forgetting (SIF) and examine whether it is deficient in psychological disorders characterized by intrusive thoughts. Specifically, we performed a focused metaanalysis of studies that have used the Think/No-Think procedure to test SIF in individuals either affected by psychological disorders or exhibiting high scores on related traits. Overall, across 96 effects from 25 studies, we found that avoiding retrieval leads to significant forgetting in healthy individuals, with a small to moderate effect size $(0.28,95 \%$ CI $[0.14,0.43])$. Importantly, this effect was indeed larger than for more anxious $(-0.21,95 \%$ CI $[-0.41,-0.02])$ or depressed individuals (0.05, $95 \%$ CI $[-0.19,0.29])$ - though estimates for the healthy may be inflated by publication bias. In contrast, individuals with a stronger repressive coping style showed greater SIF $(0.42,95 \%$ CI $[0.32$, 0.52]). Furthermore, moderator analyses revealed that SIF varied with the exact suppression mechanism that participants were instructed to engage. For healthy individuals, the effect sizes were considerably larger when instructions induced specific mechanisms of direct retrieval suppression or thought substitution than when they were unspecific. These results suggest that intact suppression-induced forgetting is a hallmark of psychological well-being, and that inducing more specific suppression mechanisms fosters voluntary forgetting.

Keywords: suppression; involuntary retrieval; cognitive control; anxiety; depression. 


\section{Introduction}

Forgetting is often regarded as a deficiency of our memory systems, where attempts to retain or retrieve information are met with failure. In particular, it has been argued to arise passively from either the temporal decay of the memory trace (Thorndike, 1913), interference from other memories that compete for retrieval (McGeoch, 1932; Underwood, 1957), or a change in context from initial encoding (Tulving, 1974).

However, under many circumstances forgetting can also be characterized as an adaptive force that shapes our memory, for instance by updating or discarding information that has become irrelevant - or even outright unwanted (Bjork, 1989; Bjork \& Bjork, 1996; for reviews, see Fawcett \& Hulbert, 2020; Nørby, 2015). Accumulating evidence suggests that such forgetting can be under intentional control: concerted attempts at preventing memories from entering awareness can subsequently make it more difficult to voluntarily retrieve these suppressed memories and eventually cause forgetting (Anderson \& Green, 2001; Hertel \& Calcaterra, 2005; see Anderson \& Hanslmayr, 2014, for review). In essence, such suppression-induced forgetting (SIF) (Anderson \& Huddleston, 2012; Hertel \& McDaniel, 2010) may serve the purpose of preventing our minds from being at the mercy of involuntary retrieval. The discarding of unwanted information may also more generally facilitate efficient cognition. For example, it supports response selection (Payne \& Sekuler, 2014) and prevents excessive information intake that may otherwise increase uncertainty (Hertwig \& Engel, 2016).

We here conducted a focused meta-analysis to scrutinize whether it is possible to foster forgetting intentionally. We were particularly interested in gauging whether such intentional forgetting may be a hallmark of psychological well-being (Benoit, Davies, \& Anderson, 2016; Depue, Curran, \& Banich, 2007; Engen \& Anderson, 2018; Joormann, Hertel, LeMoult, \& Gotlib, 2009; Visser et al., 2018) and thus be deficient in people with disorders characterized by intrusive thoughts. 
80 Intentional forgetting has been suggested to regulate our affective experience by preventing unwanted, affectively loaded memories from entering awareness. Moreover, unlike mere avoidance, it may reduce their accessibility - and possibly availability - in the long run and thus exert a persisting effect (Engen \& Anderson, 2018). Intentional forgetting may also contribute to the reappraisal of experienced events and their emotional impact by overriding, or substituting, maladaptive responses with more favorable alternatives (Hertel \& Calcaterra, 2005; Engen \& Anderson, 2018).

Conversely, a deficiency in controlling one's memories and thoughts may be at the heart of several psychological disorders (e.g., Goschke, 2014; Hertel, 1997, 1998,

90 2007; McTeague, Goodkind, \& Etkin, 2016). Perhaps most prominently, posttraumatic stress disorder (PTSD) is characterized by intrusive memories and unintentional re-experiencing (Brewin, 2014; Ehlers, Hackmann, \& Michael, 2004; Hackmann, Ehle, Speckens, \& Clark, 2004). Indeed, this feature of PTSD has been recognized as one of its defining aspects in both the Diagnostic and Statistical Manual 95 of Mental Disorders (5th ed.; DSM-5; American Psychiatric Association, 2013) and the International statistical classification of diseases and related health problems (11th ed.; ICD; World Health Organization, 2018).

The intrusiveness of memories in PTSD may result from an impaired ability to keep unwanted memories at bay (Ehlers et al., 2004; Hackmann et al., 2004). Patients 100 with this condition tend to seek help after intrusive memories are already strongly consolidated, thus highlighting the importance of understanding the retrieval processes that support the intrusions (Marks, Franklin, \& Zoellner, 2018). Similarly, intrusive negative thoughts constitute central symptoms of other affective disorders such as anxiety (Kircanski, Johnson, Mateen, Bjork, \& Gotlib, 2016) and depression 105 (Kircanski, Joormann, \& Gotlib, 2012). These intrusive thoughts have also been suggested to arise from the involuntary retrieval of previously experienced or imagined episodes (Iyadurai et al., 2018a; Visser, Lau-Zhu, Henson, \& Holmes, 2018). 

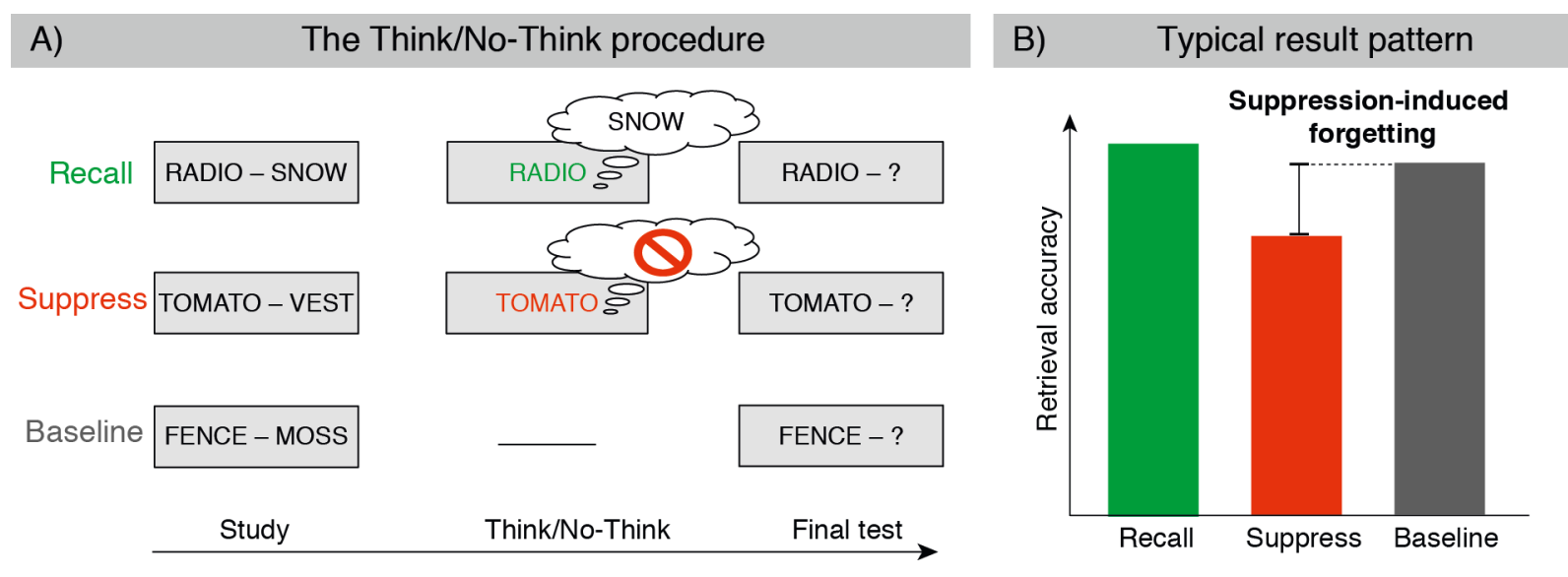

Figure 1. Panel A) Overview of the Think/No-Think procedure. In the initial study phase, participants encode associations of cues (e.g., RADIO) and targets (e.g., SNOW). They then enter the critical Think/No-Think phase, in which they repeatedly encounter most of the cues. For some of the cues (here for those presented in green), participants attempt to recall the associated targets (recall items). For other cues (here for those presented in red), their task is to prevent the associated target memory from coming to mind (suppress items). A third of the targets that they had also initially learned are not cued during this phase (baseline items). On a final test, participants are asked to remember all targets given their respective cues, irrespective of the previous instructions. Panel B) Typical retrieval accuracy on the final test. Participants are generally better or similarly capable at remembering recall than baseline targets. Critically, they are typically worse at retrieving previously suppressed than baseline targets. We refer to this finding as suppression-induced forgetting.

To examine SIF in healthy and clinical populations, we meta-analyzed studies that have employed the Think/No-Think procedure (Anderson \& Green, 2001) ${ }^{1}$. In this procedure (Figure 1), participants first learn to associate pairs of cues and targets (e.g., TOMATO - VEST), so that they can retrieve the target (VEST) upon presentation of its cue (e.g., TOMATO). Participants then enter the critical Think/NoThink phase, where they are shown a subset of the cues. For some of these cues, participants have to covertly rehearse the associated target (i.e., recall items). For other cues, participants need to actively prevent the associated target from coming

1 Note that there are also other experimental procedures that examine intentional forgetting. These include the List-Method Directed Forgetting procedure (Bjork, 1970), which has also been linked to putative inhibitory mechanisms akin to those thought to cause SIF (Bjork, 1989; Bjork \& Bjork, 1996; Anderson \& Hanslmayr, 2014; cf. Sahakyan, Waldum, Benjamin, \& Bickett, 2009). However, we focused on the Think/No-Think procedure because our aim was to assess motivated forgetting (i) at the stage of memory retrieval rather than encoding, and (ii) directed at specific items in memory rather than lists (Anderson \& Hanslmayr, 2014). 
to mind (i.e., suppress items). Each of those cues are presented several times, so to provide multiple opportunities for memory-control mechanisms to be deployed. A number of cues are not shown at all during this phase (i.e., baseline items), and serve to assess baseline memory performance in a following test phase. On that test, participants are instructed to recall each response (e.g., VEST) upon presentation of its specific cue (e.g., TOMATO), irrespective of previous instructions. Typically, participants are impaired at retrieving previously suppressed memories as indicated by worse memory accuracy for suppress than for baseline items. This finding of below-baseline memory accuracy is considered an index of SIF.

Though there has been accumulating evidence for SIF over the last 20 years (for review, see Anderson \& Huddleston, 2012; Anderson \& Hanslmayr, 2014; Wessel, Albers, Zandstra, \& Heininga, 2020, preprint), including SIF-like effects in implicit/indirect memory tests (Hertel, Large, Stuck, \& Levy, 2012; Gagnepain, Henson, \& Anderson, 2014; Hertel, Maydon, Ogilvie, \& Mor, 2018; Wang, Luppi, Fawcett, \& Anderson, 2019), this phenomenon has not universally been replicated (e.g., Algarabel, Luciano, \& Martínez, 2006; Bergström, Velmans, de Fockert, \& Richardson-Klavehn, 2007; Bulevich, Roediger, Balota, \& Butler, 2004; Mecklinger, Parra, \& Waldhauser, 2009; Wessel, Wetzels, Jelicic, \& Merckelbach, 2005). A major goal of this analysis is thus to determine the statistical significance and magnitude of the SIF effect in healthy individuals. This is particularly important to also evaluate related deficits in clinical populations.

Some of the inconsistencies in the literature may reflect important study differences with respect to the exact mechanisms that people engaged to prevent unwanted retrieval. While initial studies were somewhat agnostic regarding the employed processes (e.g., Anderson \& Green, 2001), there is now evidence for two specific suppression mechanisms. On one hand, people can prevent recall by stopping the retrieval process altogether (Benoit \& Anderson, 2012; Bergström, de Fockert, \& Richardson-Klavehn, 2009; Gagnepain et al., 2014). This mechanism, direct retrieval suppression, has been associated with an inhibitory top-down modulation of the hippocampus that originates from the right dorsolateral prefrontal cortex (Benoit \& 
Anderson, 2012; Gagnepain et al., 2014). The other mechanism, thought substitution, requires participants to retrieve an alternative memory when faced with a cue to an unwanted memory. This substitute memory then occupies the limited focus of awareness and thus prevents the unwanted memory from coming to mind (Benoit \& Anderson, 2012; Bergström et al., 2009; Hertel \& Calcaterra, 2005). Thought substitution has been associated with memory selection processes supported by the left ventrolateral prefrontal cortex (Benoit \& Anderson, 2012). Critically, both of these mechanisms have been shown to cause forgetting (Benoit \& Anderson, 2012; Bergström et al., 2009; Hertel \& Calcaterra, 2005). We will thus examine whether SIF in healthy individuals varies according to the induced suppression mechanism.

Turning to clinical populations, there is indeed evidence for impaired SIF, for example in PTSD (Sullivan et al., 2019; Waldhauser et al., 2018; Catarino, Küpper, WernerSeidler, Dalgleish, \& Anderson, 2015). However, the reliability of such a deficiency in clinical populations is still uncertain, because several studies did not directly observe impaired SIF (as compared with the respective healthy control group). Instead, these studies inferred memory control impairments from other between-groups differences that are less stringent indices of impaired intentional forgetting. These include better recall of suppress items (e.g., Hertel \& Gerstle, 2003), impaired recall of baseline items (e.g., Hertel \& Mahan, 2008), and different patterns of neural activation during the Think/No-Think phase as revealed by functional MRI (Sacchet et al., 2017).

180 To shed light on these issues, we meta-analyzed studies that compared clinical samples and sub-clinical samples (i.e., individuals displaying high scores on relevant clinical dimensions; see 2.1 for details) with healthy controls on SIF as elicited by the Think/No-Think procedure. Specifically, we predicted a significant SIF effect for healthy control groups, as well as a significant difference between healthy control groups versus clinical and sub-clinical samples.

Furthermore, we explored the effects of a few important features that might influence the magnitude of SIF. First, we assessed the impact of providing different instructions 
that are either targeted at inducing specified mechanisms (i.e., direct retrieval suppression or thought substitution) or that leave it to the participants to prevent

\section{Method}

\subsection{Search strategy and inclusion criteria}

We sought to identify all studies that had used the Think/No-Think procedure to retrieval anyway they see fit (i.e., unspecified instructions). We hypothesized that participants would benefit from instructions that induce a specific mechanism. Intriguingly, it has been suggested that depressed individuals, whose cognitive control may be deficient, could particularly benefit from a mechanism like thought substitution that aids in avoiding unwanted retrieval by providing substitute memories (Hertel \& Calcaterra, 2005).

Second, we examined whether the valence of the memories influences SIF, and whether this is especially the case for participants affected by (sub)clinical conditions. This is based on the idea that mood-congruent recall effects might modulate the effectiveness of memory control (Gaddy \& Ingram, 2014; Matt, Vázquez, \& Campbell, 1992). For instance, individuals with depression may be more prone to recall negative information, and therefore may also have a harder time suppressing it. Third, we tested whether more repetitions of a given suppress cue are associated with stronger SIF, as more repetitions provide more opportunities for successful suppression (as suggested by, e.g., Anderson \& Green, 2001; Joormann et al., 2009). Fourth, we assessed the effects of presentation time for suppress cues. With longer presentation times, the suppression effort has to be sustained for a more extended period. This has recently been shown to cause more memory intrusions (van Schie \& Anderson, 2018). We examine whether it also reduces SIF. Fifth, to inform future developments, we explored whether the effect size of SIF is sensitive to the type of material that had to be suppressed (i.e., words or pictorial material).

compare healthy groups with clinical or sub-clinical samples typically associated with 
cognitive control difficulties. We conducted our search in PubMed, Web of Science, and Google Scholar (on September 15, 2017) ${ }^{2}$, using combinations of the following search terms: Think-No Think and/or motivated forgetting, and disorders-related keywords: such as thought control ability, impulsivity, anxiety, depression, 220 dysphoria, ADHD (attention deficit hyperactivity disorder), OCD (obsessivecompulsive disorder), PTSD, schizophrenia, rumination, addiction, substance abuse, borderline, repressive coping. (The term suppression-induced forgetting produced consistently redundant results and was dropped from the search strategy). Our literature search also included key terms related to questionnaires and tasks commonly associated with the broader literature on anxiety, depression, and thought control deficits. Specifically, these were the STAI (State-Trait Anxiety Inventory), PANAS (Positive and Negative Affect Schedule), Beck Anxiety Inventory, Beck Depression Inventory, White Bear Suppression Inventory, and the Thought Control Ability questionnaire (TCAQ; Luciano, Algarabel, Tomás, \& Martínez, 2005). For exploratory purposes, we also included terms related to control and control deficits more broadly, i.e., Stop-Signal Task, N-Back, OSPAN (Operation Span), BIS-11 (Barratt Impulsiveness Scale), Rumination Response Scale, Go/No-Go, Stroop, and Flanker. In addition, we consulted two recent review articles for additional references (Hulbert, Hirschstein, Brontë, \& Broughton, 2018; Nørby, 2018), and included two studies that were published after the initial literature search had been completed (Waldhauser et al., 2018; Noreen, Cooke, \& Ridout, 2019).

Finally, we attempted to identify pertinent studies that had not been published in peer-reviewed journals. Including such studies helps providing an overall SIF effect size estimate that is less influenced by publication bias (Thornton \& Lee, 2000). In 240 August 2019, we therefore searched the ProQuest database for otherwise unpublished dissertation projects using the terms "Think/No-Think" and "motivated forgetting". In addition, in August 2019, we emailed the corresponding authors of

2 In addition, as recommended by a reviewer, we also performed searches of the ERIC and Scopus databases with the "Think/No-think" or "motivated forgetting" search terms in July 2019. However, these searches did not yield any additional paper that compared healthy with clinical or sub-clinical samples on SIF. 
relevant publications. These included the authors of the clinical Think/No-Think studies that we had identified in the literature search described above.

245 We further extended this call to corresponding authors of other papers on SIF (i.e., those not studying SIF in clinical populations) and of other papers on the related topics of Retrieval-Induced Forgetting and List-Method Directed Forgetting (as identified through PubMed and Web of Science) $(N=56)$. This procedure led to the inclusion of a doctoral dissertation that contained otherwise unpublished Think/No-

250 Think data that matched our inclusion criteria (see below) (Ryckman, 2015).

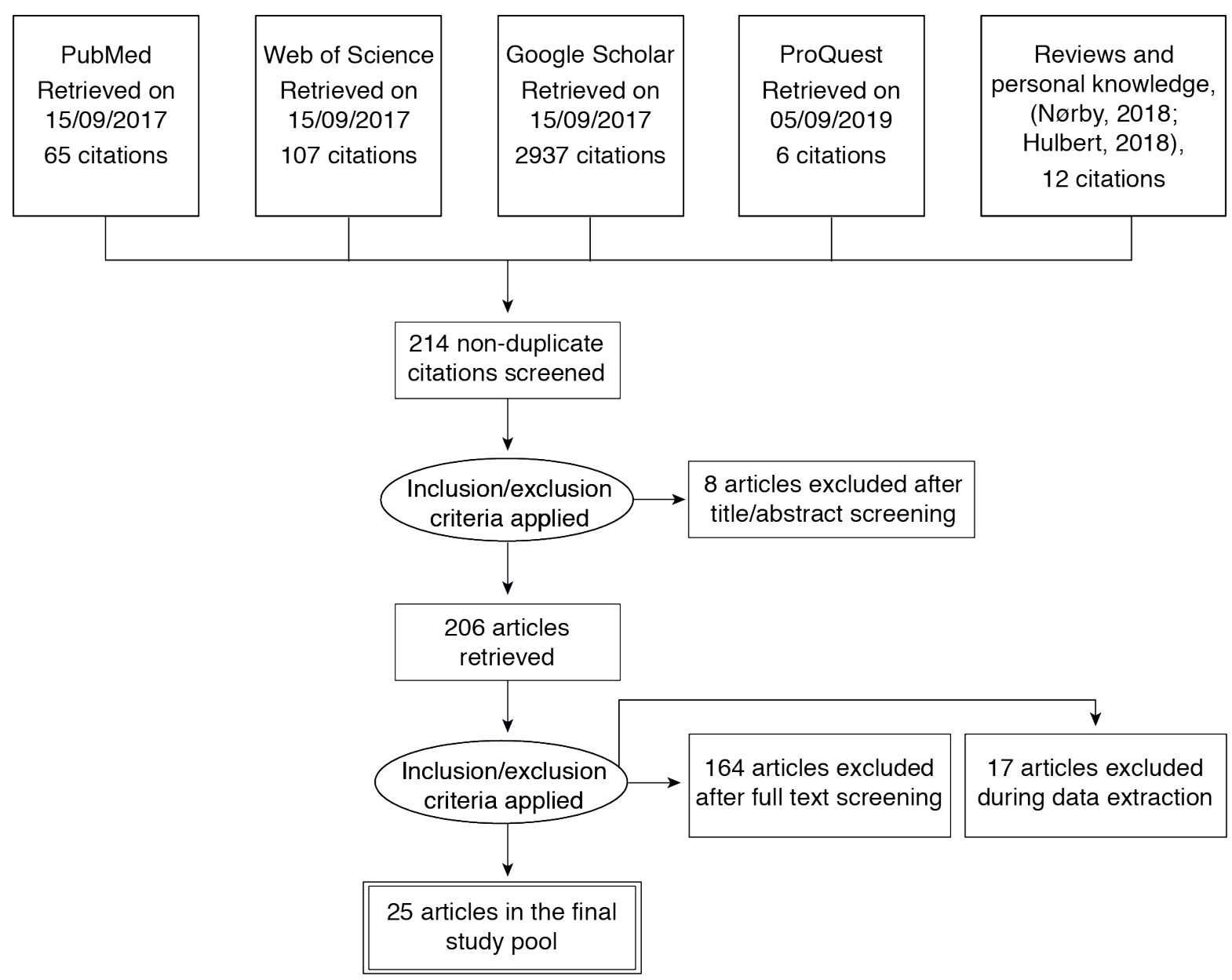

Figure 2. Schematic overview of the literature search and inclusion process. 
Moreover, if necessary, we further asked the authors of the included Think/No-Think studies for all the information required to compute effect sizes or, alternatively, for the respective data sets so that we could extract them ourselves. We thus received additional information for six studies (Depue, Burgess, Willcutt, Ruzic, \& Banich, 2010; Wessel et al., 2005; Hertel \& Gerstle, 2003; Hertel \& Mahan, 2008; Hertel \& McDaniel, 2010; Stephens, Braid, \& Hertel, 2013).

For all data that we had received directly from the respective authors, we used the newly obtained descriptive statistics instead of those extracted from the articles (many of which required direct extraction from the figures as described below). This procedure also provided the necessary information that allowed us to include the article by Stephens et al. (2013) into the study pool.

265 The inclusion procedure for the retrieved studies is summarized in Figure 2, following the recommendation of Moher, Liberati, Tetzlaff, \& Altman (2009). We included all studies that compared at least one clinical sample to a healthy control group. We also included, as sub-clinical samples, studies with groups of participants that scored high on questionnaires of clinical relevance (i.e., BDI, STAI, and RRS), or studies that split their participants into sub-clinical and control groups based on such questionnaires. We included only studies that used the Think/No-Think procedure; that reported at least one test outcome pertaining to episodic memory performance; that reported sufficient data for the meta-analysis either in text, figures, supplementary material, or that were made available in response to our request.

\subsection{Data extraction}

In total, the search yielded 214 unique entries, of which 25 entered our quantitative analysis (Table 1 ). These were coded by three of the authors (DFS, KR, and AK-M). DFS and A-KM had extensive knowledge of the SIF literature and practical expertise with the Think/No-Think procedure; KR had previous experience with literature search for meta-analyses. 
In a first step, DFS and KR jointly recorded recall performance (for "same probe" tests as opposed to "independent probe" tests; see section 4.1.) for baseline and suppress items of each group, as well as five potential moderators of the effect (see below). They also coded the nature of the clinical or sub-clinical condition.

In a second step, to ensure the reliability of the data extraction, a third author (AKM) independently coded all of the information. A-KM and DSF then examined their inter-rater agreement and reached full consensus with respect to the moderators and clinical condition. For many of the included studies, the critical mean values and measures of dispersion were only provided in plots (Table 1 ). In these cases, they manually extracted these values using WebPlotDigitizer (Rohatgi, 2017), which has been shown to yield high inter-coder reliability (Drevon, Fulsa, \& Malcolm, 2017).

Indeed, our two sets of coding also only yielded minor differences. We thus obtained high inter-rater reliability on those measure, in terms of high criterion-referenced reliability, a case of intraclass correlation coefficient (ICC; McGraw \& Wong, 1996) suitable for comparing our coding. Indeed, the lowest ICC was 0.98 , and all coefficients were significantly different from 0 (all $p<.001$ ). We thus deemed it appropriate to reach a consensus by averaging the two respective sets of values.

Several studies reported multiple, non-independent measures of SIF. These included retrieval accuracy on different test formats and multiple ways of rating the quality of the retrieved memories. Similarly, some studies employed within-subject manipulations of, e.g., the number of repetitions during the Think/No-Think phase or the valence of the suppress items. They therefore provided multiple estimates of SIF (i.e., one for each level of the within-subject manipulation). In general, we included 305 all the non-independent SIF measures. This was always the case for effects related to moderators of interest (e.g., SIF from different sets of suppress items each characterized by a different emotional valence). For studies that employed experimental manipulations other than those identified as moderators of interest (e.g., homograph or non-homograph stimuli in Hertel \& McDaniel, 2010), we coded 310 all the non-independent SIF measures unless the authors had only provided 
aggregate data across levels of the manipulation ${ }^{3}$. Similarly, whenever task features were manipulated between-groups, we included all independent SIF effects, as long as it was possible to distinguish performance of healthy from that of clinical and subclinical participants. For example, when different samples of participants suppressed

315 either positive or negative items, we included both of the resulting independent effect sizes. For two studies (Kim, Yi, Yang, Lee, 2007; Kim, Oh, Kim, Sim, \& Lee, 2013), we could not obtain any dispersion measures for memory performance. We estimated the missing standard deviations (see Higgins \& Green, 2011, p. 485) by calculating the respective means of the standard deviations of the other included studies, weighted by their respective sample sizes (for this procedure, we excluded the few instances where outcomes were not reported in percentage form; Catarino et al., 2015; Küpper, Benoit, Dalgleish, \& Anderson, 2014).

In addition, we coded for the five potential moderators of SIF. First, we coded the nature of the instructions given to participants to prevent retrieval (direct retrieval 325 suppression, thought substitution, or unspecified). One study had different participants assigned to either unspecified or thought substitution instructions (Noreen \& Ridout, 2016a), but did not provide separated SIF results as a function of both, instructions and group. For each group, we therefore took the SIF effects combined across the two instruction conditions and marked them as unspecified.

330 Second, we coded the valence of the stimulus material (for the suppress targets only) as either neutral, positive, negative, or mixed (i.e., when the only reported effect sizes were combined across different valence levels). When studies comprehensively reported SIF for different valence categories assigned to the same participants (e.g.,

3 With this approach, we assess the robustness of SIF across many different manipulations and means of quantifying SIF. In Appendix 1, we provide two complementary random-effects models (one for the healthy and one for the (sub)clinical samples) that only include the single independent effect size of each study that constitutes the condition or measurement most typically used to assess SIF in the extant literature (for justifications of this approach, see Card, 2012, pp. 192-193; Cooper, Hedges, \& Valentine; 2019, p. 282; Higgins and Green, 2011, Chapter 3; Lipsey and Wilson, 2001, p. 125). These models thus provide an estimate of the presumably strongest manipulations (e.g., the greatest rather than fewer suppression repetitions). (These models further allow for a comparison with our initial preprint available at PsyArXiv doi: 10.31234/osf.io/5wynm). 
for neutral, negative, and positive memories in Marzi, Regina, \& Righi, 2014; neutral and negative in Sacchett et al., 2017; Zhang, Xie, Liu, \& Luo, 2016), we generally included the effect size related to each condition, where available. Finally, for one study (Dieler, Herrmann, \& Fallgatter, 2014) we coded SIF for negative items only, because its analysis of group differences (low vs. high anxiety) did not include neutral items.

340 Third, we coded the repetitions of suppress items, i.e., the number of times that participants encountered each cue in the Think/No-Think phase. One study reported a SIF effect averaged across two conditions with two and eight repetitions (Noreen \& Ridout, 2016a). We here coded the average (five) as the number of repetitions associated with that effect size, as we could not obtain the data set to disentangle the two.

Fourth, we coded the duration for which cues remained on the screen during the Think/No-Think phase, and, fifth, the material of stimuli that participants had to suppress (i.e., words or pictorial material). One study (Stephens et al., 2013) that had examined the recall of autobiographical memories was also coded as words.

\subsection{Statistical analysis}

Our main focus was twofold: assessing the statistical significance and magnitude of SIF in healthy individuals and determining whether SIF is indeed reduced in (sub)clinical samples characterized by intrusive thoughts and deficits of cognitive control. We therefore computed a series of meta-analyses in R 3.6.1 ( $R$ Development Core Team, 2008) with the package metafor 2.1.0 (Viechtbauer, 2010). 
We first clustered studies based on clinical and sub-clinical conditions. Specifically, we grouped the clinical samples with respect to the psychiatric taxonomy of the DSM (5th ed., American Psychiatric Association, 2013) (i.e., anxiety, depression) (note that we grouped PTSD with anxiety, given that this is an often co-occurring feature 365 of this disorder) or the similarity of their defining characteristic (i.e., high repressive coping style). We added the sub-clinical samples according to their relatedness along the psycho-pathological continuum (e.g., we combined depressed mood with major depressive disorder) (Table 1 ).

We thus identified a depression cluster (20 effect sizes from 11 studies, including 370 major depressive disorder, dysphoria, and rumination, $N=324$ (sub)clinical participants), an anxiety cluster (nine effect sizes from five studies, including high trait anxiety, generalized anxiety disorder (GAD) and PTSD, $N=90$ (sub)clinical participants), and a repression cluster (nine effect sizes from three studies, including high repressive coping, $N=78$ (sub)clinical participants). Repression, unlike the other

375 (sub)clinical clusters, has previously been linked to a stronger ability to prevent retrieval, and we thus expected greater SIF for this cluster (Hertel \& McDaniel, 2010).

We assigned the remaining effect sizes to a mixed cluster (ten effect sizes from six studies, with $N=243$ (sub)clinical participants). This cluster included one study each on alcohol abuse, ADHD, schizophrenia, low thought control ability (as measured by 380 the TCAQ; Luciano et al., 2005; greater scores on the TCAQ are negatively associated with both anxiety and depression as well as obsessive-compulsive disorder; Williams et al., 2010), dissociative disorders (as measured by the Dissociative Experiences Scale, DES; Bernstein \& Putnam, 1986), and high neuroticism (as measured by the Neuroticism subscale of the Eysenck Personality Questionnaire - Revised, EPQ-R; 385 Eysenck, Eysenck, \& Barrett, 1985). The high heterogeneity of samples included in the mixed cluster hinders meaningful comparisons with the other, more clinically defined clusters. We therefore only examine it on its own and refrain from any comparison. Finally, the effect sizes of all control groups were combined in one healthy cluster (48 effect sizes, $N=687$ healthy participants). 
390 We computed all the effect sizes as the standardized mean change score between baseline and suppress items (as implemented in the escalc function; measure set to SMCC), using the extracted means and standard deviations. However, this method requires an estimate of the correlation between baseline and suppress items, which was not reported in the surveyed literature. We thus estimated the Spearman correlation as $r=.3$, based on data from our group and on the studies for which we were able to obtain the respective datasets (Wessel et al., 2005; Hertel \& Gerstle, 2003; Hertel \& Mahan, 2008; Hertel \& McDaniel, 2010; Stephens et al., 2013). The original correlation coefficient was retained for these data sets. (Note that additional sensitivity analyses, using alternative correlation coefficients of $r=.1$ and .6 , yielded the same conclusions with respect to our main hypothesis. Accordingly, only results obtained with an assumed correlation of .3 will be reported. Two studies (Catarino et al., 2015; Küpper et al., 2014) had employed three fairly different measures of SIF. For these, we used correlation coefficients of three unpublished data sets from our group for which we had used the same measures.

405 We then performed a random-effects meta-regression (Hedges \& Olkin, 1985) of the SIF effect sizes, grouped by cluster ( $N=1588$ participants; 96 effect sizes from 25 studies). To account for the correlation between some of the effect sizes, we used a multi-level random-effects (MLRE) model (Konstantopoulos, 2011), with random effects (intercepts) for both the sample (i.e., sampleID) and study (i.e., studyID)

410 from which the data were derived, with the former nested within the latter. In an earlier iteration of the analysis, we had fitted four-level models including an additional random effect for each of the individual effect sizes. However, there was no advantage in this approach since that variable explained little to no variance, and ultimately did not yield any difference. We therefore removed it in favor of a simpler model structure.

We then estimated robust confidence intervals for the effect sizes with the robust.rma.mv function, with study identifier (studyID) as the clustering variable used for constructing the sandwich estimator (see Hedges, Tipton, \& Johnson, 2010). This approach allowed us to account for violations in the independence assumptions 
due to multiple effect sizes arising from the same participants, which were frequent in our data set; and, more generally, for the correlation between effects within each study. We used this approach for all of the following analyses.

To assess whether this analysis was more informative than a simpler random-effects meta-analysis of all effect sizes irrespective of any distinction by cluster, we used 425 Akaike's Information Criterion (AIC; Akaike, 1998) with small-sample correction $(A I C C)$, transformed to conditional probabilities for each model (Wagenmakers \& Farrell, 2004). The resulting AIC weights (AICW) thus provide evidence for the relative fit of the two compared models to the data (note that all AICW for a set of models sum up to 1 ).

430 We computed $A I C C$ and $A I C W$ using the fitstats (from the metafor package) and akaike.weights (from the qpcR package; Spiess, 2018) functions in $R$. In general, we fitted our models using Restricted Maximum Likelihood (REML) as opposed to the Maximum Likelihood (ML) method due to ML's bias in variance components' estimates. However, AIC is not suitable for comparing sets of models that have been 435 fitted with REML and differ in their fixed effects structures. The model comparisons were thus based on models that were refitted using ML. We report the best fitting model.

We complemented the meta-analyses of the healthy versus (sub)clinical samples with a series of further moderator analyses. We performed separate analyses for each of 440 the five moderators (instructions, valence, repetitions, duration, and material). These analyses were carried out on a reduced sample of studies, combining the anxiety and depression clusters in a single emotional disorders group (e.g., Goodwin, 2015). These choices were due to the relatively small pool of studies that contributed to each level of the moderators and to achieve a reasonable homogeneity of the included 445 clinical samples. The moderator analyses thus do not include the repression cluster, due to its - expected - opposite effect on intentional forgetting (Hertel \& McDaniel, 2010), and the mixed cluster, due to the diversity of the samples' (sub)clinical characteristics. Overall, this approach, with group (healthy versus emotional 
disorders) rather than cluster, thus served to increase the power of the analyses while maintaining theoretical consistency.

For each moderator analysis, we compared the AICW of a model that included a moderator*group interaction with that of a moderator+group model that only included main effects. We report only the best fitting model, or, in cases where models performed similarly, the simpler one. We had planned to carry out these five moderator analyses for theoretical reasons, and they were further motivated by the high heterogeneity consistently observed in our models. Therefore, we expected that the chosen moderators might explain part of this heterogeneity.

For all the reported models, we evaluated heterogeneity across samples by calculating the 95\% Prediction Interval (PI; IntHout, Ioannidis, Rovers, \& Goeman, 2016). The PI indexes the range of effects expected from new samples similar to those included in the analysis. Heterogeneity was also tested with Cochran's $Q$ (Cochran, 1954), where a significant outcome rejects the null hypothesis that all the included studies evaluated the same effect ( $Q E$ was used for models that included moderators). We further examined $I^{2}$, which indicates how much of the overall variation across studies is due to heterogeneity as opposed to mere chance (Higgins, Thompson, Deeks, \& Altman, 2003). Specifically, we used a generalized form (Nakagawa \& Santos, 2012) that allowed us to quantify such proportions separately for higher-level ( $I^{2}$ studyID, for studyID) and nested $\left(I^{2}\right.$ sampleID, for sampleID) random factors.

470 Meta-analyses are susceptible to publication bias, i.e., the inflation or otherwise distortion of effect size estimates due to selective reporting of favorable study outcomes (Thornton \& Lee, 2000) and other forms of questionable research practices (Renkewitz \& Keiner, preprint). In particular, in the context of the present metaanalyses, there could be a bias for reporting experiments that yielded a significant 475 SIF effect for the healthy individuals. Alternatively, there could be a bias for publishing studies that did not find significant SIF in the (sub)clinical groups. 
Ideally, this problem would be mitigated by including all unpublished studies, assuming that they were not published for exactly these biases. However, we only retrieved three such experiments (Ryckman, 2015).

Therefore, to further gauge these biases, we used contour-enhanced funnel plots to display each study's effect size against its precision as indexed by the standard error (Peters, Sutton, Jones, Abrams, \& Rushton, 2008). These plots are centered at zero, and display areas of statistical significance. This, in turn, allows for easier visual detection of publication bias due to exclusion of studies that yielded non-significant results. We plotted effect sizes separately for healthy individuals and (sub)clinical groups.

We then used Egger's regression test (e.g., Peters, Sutton, Jones, Abrams, \& Rushton, 2006; Egger, Smith, Schneider, \& Minder, 1997) to formally assess funnel plot asymmetry as an indicator of publication bias (with $p<0.1$ as the critical value, following the recommendation of Egger et al., 1997). Because this test is not yet implemented for MLRE models ${ }^{4}$, we performed it by re-estimating each model (healthy individuals or (sub)clinical groups) with the inclusion of a moderator coding for the standard error of the effect sizes. A significant deviation from zero in the intercept of this meta-regression would indicate that the relationship between precision and size of the studies is asymmetrical, and thus biased (Sterne \& Egger, 2005).

Because the sensitivity of meta-analytic estimates is also vulnerable to outliers in the study pool, we also evaluated the included studies for influential cases, based on Cook's distance (cooks. distance.rma.mv, clustered by studyID). This is a leave-oneout diagnostic measure (available in metafor) that is suitable for data sets with a

4 For the same reason, we could not adjust for publication bias using the trim-and-fill procedure (Duval \& Tweedie, 2000). However, in Appendix 1, we additionally perform this procedure for the simpler random-effects models that are based on only the single effect sizes from each study that are derived from the most typical measures and manipulations. Appendix 2 provides further simple random-effects models using fill-and-trim correction based on the average effect sizes from each study. Note that we apply these methods irrespective of the non-significant Egger's test of the main analysis. 
multi-level structure and for the robust estimation of confidence intervals (Cook \& Weisberg, 1982; Viechtbauer \& Cheung, 2010). We thus compared the model that was most informative in respect to our hypotheses - the MLRE meta-regression with studies grouped by cluster - fitted with and without studies that appeared to be highly

505 influential as indicated by a Cook's distance greater than 1 (Hair, Anderson, Tatham, \& Black, 1998).

\section{Results}

\subsection{Suppression-induced forgetting in healthy versus (sub)clinical samples}

First, we assessed whether a model using the cluster moderator (i.e., healthy, anxiety, depression, repression, and mixed) would provide a better fit to the data than a simpler model with effect sizes classified as just healthy or (sub)clinical, or than the basic null model without any moderator. Indeed, this was the case, with the cluster model ( $A I C W_{\text {cluster }}=.72$ ) being approximately 2.8 times more plausible than 515 the simple model $\left(A I C w_{\text {simple }}=.26\right)$ and 36 times more plausible than the null model $\left(A I C w_{\text {null }}=.02\right)$. The cluster model also displayed the lowest overall heterogeneity, $I^{2}=67.85 \%$. Overall, these results support using the cluster model as the benchmark for testing our two main hypotheses.

Importantly, the overall effect of the moderator cluster was significant, $F_{(4,20)}=$ $18.93, p<0.001$. There was a significant small-to-moderate SIF effect of $0.28,95 \%$ CI $[0.14,0.43], 95 \%$ PI $[-.38, .95], p<.001$ for the cluster of all healthy samples. By comparison, the anxiety cluster displayed a small significant effect in the opposite direction (indicating significantly higher recall of suppress than baseline items), with an estimate of $-0.21,95 \%$ CI $[-0.41,-0.02], 95 \%$ PI $[-0.89,0.47], p=.036$. The depression cluster did not show evidence in support of any effect, with an estimate of $0.05,95 \%$ CI $[-0.19,0.29], 95 \%$ PI $[-0.64,0.74], p=.662$. The effect for the mixed cluster was in the direction of SIF, but failed to reach significance, with an estimate of $0.17,95 \%$ CI $[-0.09,0.43], 95 \%$ PI $[-0.53,0.87], p=.188$. However, 
the repression cluster yielded a significant effect, with an estimate of $0.42,95 \% \mathrm{CI}$ $[0.32,0.52], 95 \%$ PI $[-0.23,1.08], p<.001$. As shown in the preceding paragraph, there was a high amount of heterogeneity, which was also significant, $Q E_{(91)}=$ $251.92, p<0.001$.

\section{Greater suppression-induced forgetting in the healthy cluster compared to the anxiety and depression clusters}

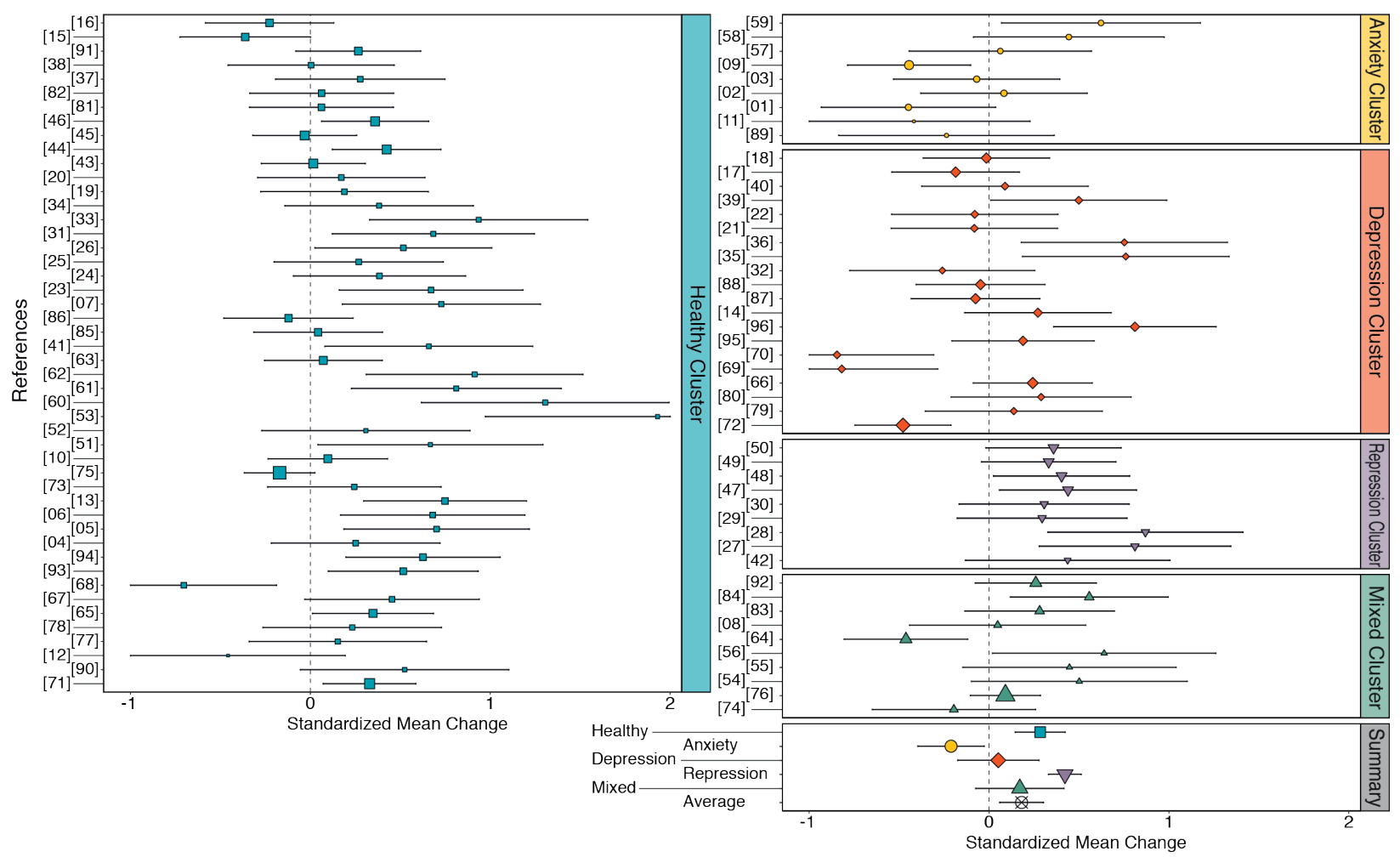

Figure 3. Forest plot of all effect sizes grouped by cluster. Standardized mean changes with change score standardization and $95 \%$ confidence interval, as a function of clinical cluster. References point to the corresponding study IDs in Table 1 . Symbols for individual effect sizes are sized proportionally to the respective sample sizes. Symbols at the bottom display the meta-analytic effect sizes from the meta-regression models and the overall effect size from the random-effects model (not sized proportionally to sample sizes).

540 Comparisons of the individual clusters displayed a significant difference for the healthy compared to the anxiety cluster, $-0.50,95 \%$ CI $[-0.65,-0.34], p<.001$, and the depression cluster, $-0.23,95 \%$ CI $[-0.45,-0.01], p=.039$. Therefore, the results corroborate our hypothesis of significant SIF in healthy individuals and of impaired SIF in clinical samples associated with emotional disorders (Figure 3). Finally, there 
545 was significantly greater SIF for the small sample of effect sizes arising from the repression cluster, compared to the healthy cluster, with a difference of $0.14,95 \%$ CI $[0.05,0.22], p=.003$.

Because the cluster model still exhibited high heterogeneity, we next investigated whether influential studies or our choice of correlation coefficients might have driven

550 the results. We thus followed an identical approach to the one described in the previous section. Here, Cook's distance indicated one particularly influential study (Marzi et al., 2014) that exceeded the set threshold of 1 . Refitting the cluster model without these effect sizes (90 effect sizes rather than 96) yielded comparable results. Furthermore, heterogeneity was still significant, $Q E_{(83)}=219.09, p<0.001$, and not

555 lower than before, $I^{2}=65.06 \%$, although more evenly spread between the random factors $\left(I^{2}{ }_{\text {studyID }}=37.70 \%, I_{\text {sampleID }}^{2}=27.36 \%\right)$. In light of these results, we chose to keep this study in the pool for the subsequent analyses.

\subsection{Moderators analysis}

560 Though the meta-analysis provided evidence for significant SIF in the general population, the included studies varied widely in the mechanism that individuals were instructed to adopt to prevent unwanted retrieval, as well as with respect to the material, valence, repetitions, and duration of the suppress items. For each moderator model, we first checked whether a model including group (healthy vs. emotional disorders) and the respective moderator provided a better fit to the data with the interaction of these factors or, more simply, with their linear combination only. Because the emotional disorders group combined the depression and anxiety clusters, we first verified that it also showed lower SIF than the healthy group. This was the case, with a significant difference of $-0.29,95 \%$ CI $[-0.48,-0.10], F_{(1,14)}=$ $10.9781, p=.005$. 


\subsubsection{Instructed mechanism}

There was a slight advantage for the instructions*group compared to the instructions+group model, with the former $\left(A I C w_{\text {instructions }} *\right.$ group $\left.=0.76\right)$ being approximately 3.2 times more plausible than the latter $\left(A I C W_{\text {instructions+group }}=0.24\right)$. Therefore, we reported the instructions*group model (Table 2 ).

The instructions moderator significantly differentiated between memory control mechanisms, though only in healthy individuals. For these samples, direct retrieval suppression displayed a medium SIF effect of 0.66 , with $95 \%$ CI $[0.42,0.90], 95 \%$ PI $[-0.02,1.34], p<.001$. The SIF effects for thought substitution was also significant, with $0.59,95 \%$ CI $[0.40,0.77], 95 \%$ PI $[-0.08,1.25], p<.001$. However, the SIF effect of unspecified instructions was not significant, with $-0.02,95 \%$ CI [$0.29,0.26], 95 \%$ PI $[-0.71,0.68], p=.902)$.

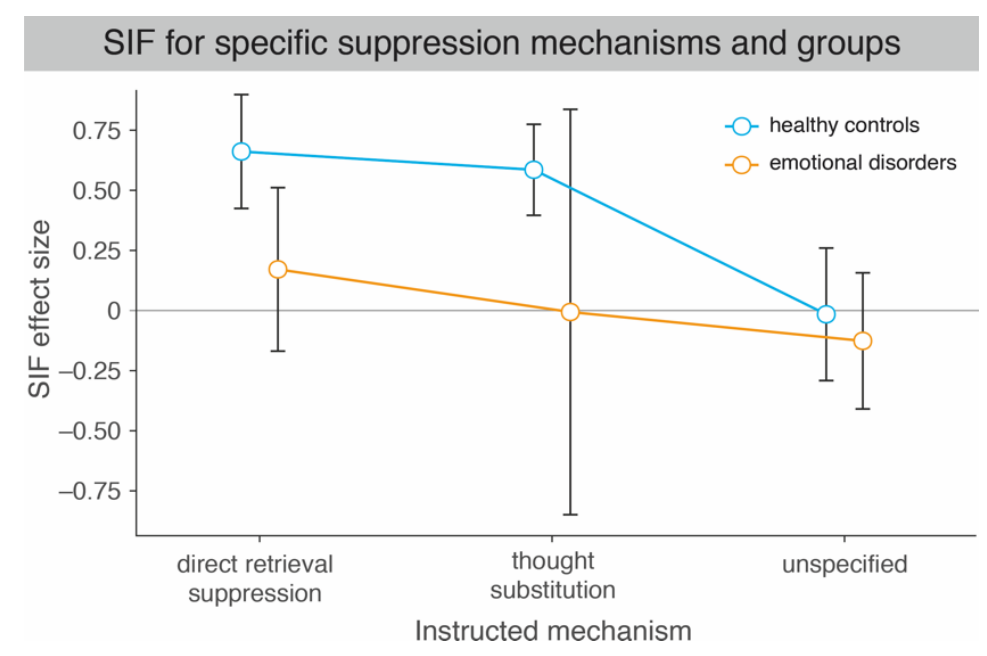

Figure 4. Average SIF effect size for instructed mechanisms, separately for the healthy and emotional disorders groups. Standardized mean changes with change score standardization and $95 \%$ confidence interval, as a function of group and instructions. Blue circles denote values for healthy groups, whereas orange circles indicate values for (sub)clinical samples with emotional disorders.

In the (sub)clinical individuals, by contrast, none of the instructed mechanisms were significant. Direct retrieval suppression displayed a small non-significant SIF effect of 0.17 , with $95 \%$ CI $[-0.17,0.51], 95 \%$ PI $[-0.55,0.90], p=.288$. SIF for thought substitution was also not significant, with an estimate of $-0.01,95 \%$ CI $[-0.85,0.84]$, 
95\% PI [-1.06, 1.53], $p=.987$. Unspecified instructions yielded a numerically reversed, albeit also not significant, SIF effect of $-0.13,95 \%$ CI $[-0.41,0.16], 95 \%$ PI $[-0.83,0.57], p=.344)$.

Notably, for healthy individuals, the SIF effect for direct retrieval suppression was significantly higher than the one for unspecified instructions, with a difference of 0.68 , $95 \%$ CI $[0.31,1.04], p=.002)$. This was also the case for the comparison of thought substitution and unspecified instructions, with a difference of $0.60,95 \%$ CI [0.28, $0.92], p=.002$. The effects for direct retrieval suppression and thought substitution were quite similar to each other, with a negligible non-significant difference of 0.08 , $95 \%$ CI $[-0.23,0.38], \mathrm{p}=.589)$ in favor of the former. The results thus corroborate the importance of instructing a specific mechanism to elicit SIF (Figure 4A). However, none of these comparisons were significant for the emotional disorders cluster (all $p$ $<0.165)$.

\subsubsection{Material}

The material*group model was very similar to the material+group model, with the former $\left(A I C w_{\text {material*group }}=0.46\right)$ being about as plausible as the latter $\left(A I C w_{\text {material+group }}\right.$ $=0.54)$. We thus decided to focus on the simpler model for the material moderator 615 (Table 3).

For the healthy group, results revealed a significant SIF for pictorial material, with a small-to-moderate effect of $0.42,95 \%$ CI $[0.09,0.75], 95 \%$ PI $[-0.39,1.23], p=$ .016), and a trend for words, with a small effect of $0.21,95 \%$ CI $[-0.02,0.44], 95 \%$ PI $[-0.57,0.99], p=.067$ ). (The same analysis on all healthy control samples from the study pool, i.e., not just those from the studies on emotional disorders, yielded significant SIF of $0.17,95 \%$ CI $[0.02,0.31], 95 \%$ PI $[-0.49,0.82], p=.026)$. SIF 
for pictorial material was twice as large as for words, though not significantly different, $0.21,95 \%$ CI $[-0.18,0.61], p=.264$. In addition, as expected, the average effect was significantly smaller for the emotional disorders group (Table 3 ).

\section{Table 3 about here ----}

\subsubsection{Valence}

The valence*group model did not improve on the simpler valence+group model, with the former $\left(A I C W_{\text {valence }}\right.$ group $\left.=0.10\right)$ in fact being 9 times less plausible than the latter $\left(A I C w_{\text {valence+group }}=0.90\right)$. We thus decided to focus on the model without an interaction for the valence moderator (see Table 4).

In terms of different valences of suppress stimuli, in healthy groups, the neutral valence condition displayed a small yet statistically significant SIF effect of 0.33 , with $95 \%$ CI $[0.04,0.62], 95 \%$ PI $[-0.55,1.27], p=.029$. Likewise, the negative valence condition was significant with an estimate of $0.29,95 \%$ CI $[0.18,0.56], 95 \%$ PI [$0.48,1.06], p=.039$. The positive valence condition was relatively similar to the neutral condition, but not significant, with an estimate of $0.36,95 \%$ CI [-0.19, 0.91], $95 \%$ PI $[-0.55,1.27], p=.177$. Instead, the mixed condition exhibited a negligible 640 SIF effect, which was not significant, with an estimate of $0.08,95 \%$ CI $[-0.22,0.39]$, $95 \%$ PI $[-0.70,0.87], p=.557$. As before, the average effect was significantly smaller for the emotional disorders group (Table 4). However, none of the comparisons were significant (all $p \geq 0.154$ ). 


\subsubsection{Repetitions}

In terms of $A I C C$, the repetitions*group ( $A I C w_{\text {repetitions }}{ }_{\text {group }}=0.36$ ) model was slightly inferior to the repetitions+group model $\left(A I C W_{\text {repetitions }+ \text { group }}=0.64\right)$. Therefore, we report the simpler model without an interaction for the repetitions moderator (Table $5)$. However, the main effect of repetitions was not significant, $F_{(1,13)}=0.59, p=$ 655.456 , providing no evidence that the amount of repetitions (within the included range) influences SIF.

\section{Table 5 about here ----}

\subsubsection{Duration}

The duration*group model $\left(A I C W_{\text {duration } * \text { group }}=0.40\right)$ was approximately 1.5 times worse than the duration+group model $\left(A I C W_{\text {duration+group }}=0.60\right)$. Therefore, we report the model without an interaction for the duration moderator (Table 6 ). The main effect of duration was not significant, $F_{(1,13)}=1.14, p=.306$, and thus we did not find evidence for a role of duration of the suppress stimuli on SIF.

Table 6 about here ----

\subsection{Publication bias analysis}

For the effect sizes arising from healthy individuals only, the contour-enhanced funnel plot exhibited some apparent degree of right-skewness (Figure 5a). However, many of the effect sizes fell within the areas of non-significance, suggesting a lighter bias. Indeed, Egger's regression for this set of effect sizes was not significant, with $p=$ .182, and thus did not provide evidence for an asymmetrical relationship between the precision and effect size of the studies. 
For the effect sizes arising from (sub)clinical samples, points in the contour-enhanced funnel plot were quite evenly scattered around the meta-analytic effect size estimate (Figure 5b). As before, Egger's regression for this set of effect sizes was not 680 significant, with $p=.409$.

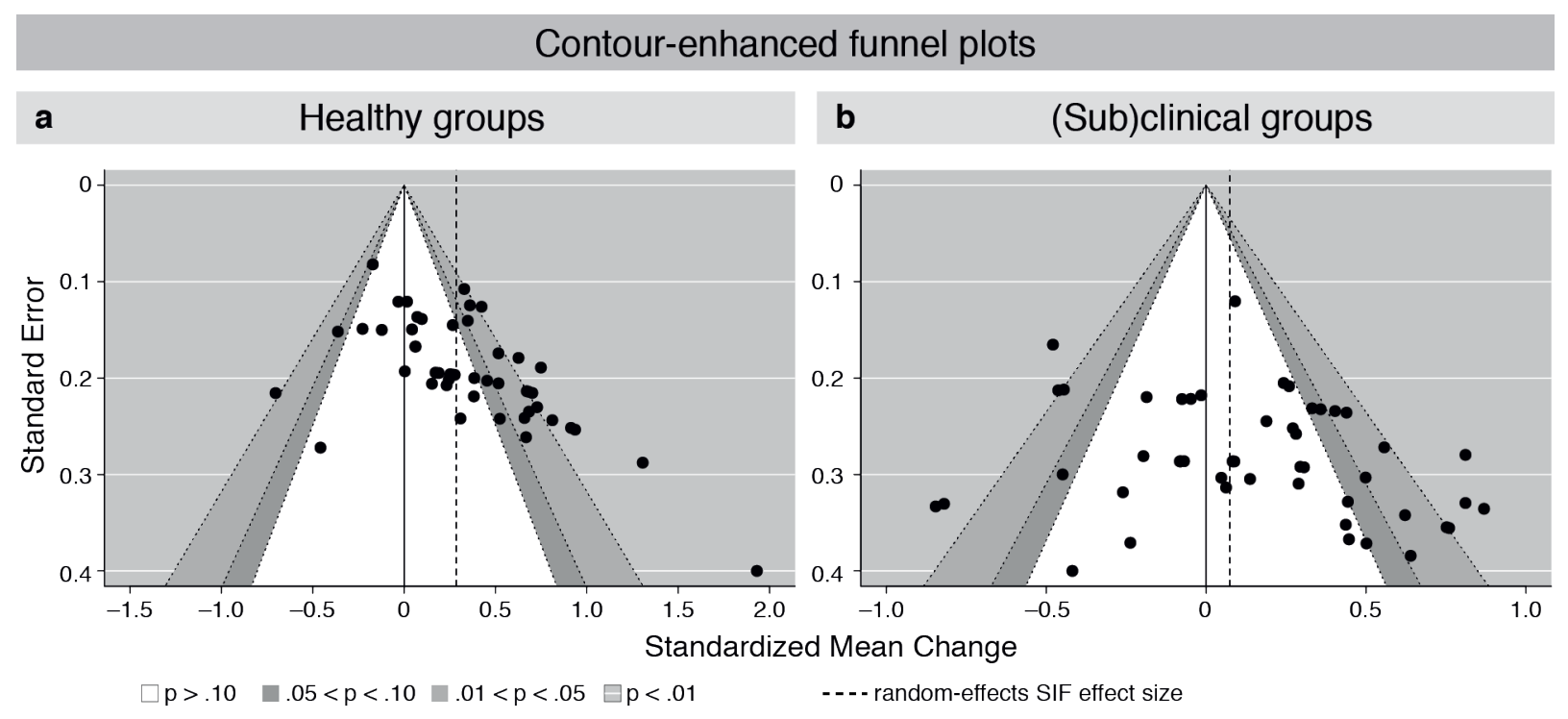

Figure 5. Contour-enhanced funnel plots of healthy (panel a) and (sub)clinical (panel b) samples displaying individual effect sizes (black circles). The black dashed lines indicate the estimated effect sizes. As indicated in the legend, each background color indicates a different area of significance within which a study may fall into: white for $p>.10$, dark gray for .05 $<<<.10$, gray for $.01<p<.05$, and light gray for $p<.01$.

\section{Discussion}

In a series of meta-analyses, we set to examine two questions: (i) whether preventing retrieval can cause forgetting and (ii) whether such suppression is deficient in individuals with psychological disorders that are characterized by intrusive thoughts. We therefore focused on studies employing the Think/No-Think procedure that compared the SIF effect between healthy control groups and relevant clinical and sub-clinical samples. In the following, we will first discuss the replicability, effect size, mechanisms, possible causes, and moderators of SIF in the general population. 
We will then turn to the evidence for impaired SIF in patient populations and discuss the implications of the results for theorizing about memory suppression as a beneficial coping mechanism.

\subsection{Significant suppression-induced forgetting in healthy adults}

Our analyses of the healthy individuals demonstrated a significant albeit small-tomoderate effect size. Inspection of the contour-enhanced funnel plots suggested some degree of publication bias, though this was not corroborated by Egger's regression test (though see 4.3). We further examined publication bias for SIF by performing trim-and-fill corrections on two complementary simple random-effects models (Appendices 1 and 2) (though see section 4.3). One model was based on the average effect sizes of each study (Appendix 2). For this model, the penalty of the trim-and-fill procedure was - surprisingly - greater when including unpublished studies that themselves had reported null results or even significant reversals than when just including published studies. The former analysis yielded an effect at the trend level only, though the latter remained significant. The other model (Appendix 1) was based on only the single independent effect sizes of each study that constituted the conditions or measurement of SIF that are most common in the literature. It thus presumably provides an estimate of the strongest manipulations (e.g., the greatest number of suppression repetitions; Anderson \& Green, 2001). This effect remained significant following trim-and-fill. Overall, the results thus corroborate that memory suppression can induce forgetting.

The healthy participants in the included studies were typically matched to the respective clinical sample on demographic measures. As a corollary, individuals of the control groups were closer to a community sample than what is usually realized in psychology experiments. We therefore suggest that the results of this analysis may be fairly generalizable to a wider population. For the same reason, however, we might have underestimated the upper boundary of the effect size that could be achieved by high functioning, young adults. 
At the same time, it is also possible that we underestimated its lower boundary, because the control individuals were typically selected to lack the clinical features that had been of interest to the respective study. Therefore, the control groups may be mentally healthier - and therefore more adept at controlling unwanted memories - than a random sample of the general population.

Across the meta-analyses, we also examined features that might influence SIF in healthy individuals and in the emotional disorders of anxiety and depression. Overall, we did not find a substantial contribution of valence, repetitions, or duration of suppression attempts towards the magnitude of SIF. These null findings may be a consequence of the overall little variance in levels of the moderators (in particular for repetitions and duration) (see also section 4.3). However, we found that SIF was numerically greater for pictorial material than for words. This finding is encouraging, given that suppressing pictures, with their greater complexity, may be more similar to suppressing autobiographical memories and thus possess higher ecological validity

740 (e.g., Stephens et al., 2013). We note, though, that there are also a number of methodological differences between studies using words versus pictures. For example, the latter tend to use more fine grained dependent variables rather than simple binary accuracy measures, which may make the former more sensitive for assessing subtle forgetting effects. However, SIF was neither significant for pictorial 745 material nor for words in the (sub)clinical samples.

Most notably, in healthy samples, there were substantial differences in SIF due to task instructions. Though all included studies formally used the Think/No-Think procedure to assess intentional forgetting, they differed with respect to whether they left it to the participants to find possible solutions to prevent retrieval or whether 750 they prescribed a specific mechanism (either direct retrieval suppression or thought substitution). In healthy participants, SIF was significantly greater under direct retrieval suppression and thought substitution instructions compared to unspecified instructions. 
These results thus clearly indicate that it is essential for future Think/No-Think studies to provide specific instructions. Indeed, Hertel and Calcaterra (2005) had previously provided evidence for stronger SIF when participants were using thought substitution rather than following unspecified instructions. In general, unspecified instructions require participants to first find possible solutions to prevent involuntary retrieval. They may also lead participants to alternate between a multitude of suppression mechanisms throughout the procedure. Both of these may diminish the efficacy of suppression and thus weaken the degree of SIF.

Suppression mechanisms may not only differ in the underlying neuro-cognitive processes (e.g., Benoit \& Anderson, 2012; Bergström et al., 2009), but also in the manner that they induce subsequent forgetting. The prominent inhibitory account of memory control suggests that suppression attempts lead to the recruitment of inhibitory processes that directly target and weaken the avoided memory trace (Anderson \& HansImayr, 2014; Detre, Natarajan, Gershman, \& Norman, 2013). As a consequence, the targeted memories should become less available in subsequent retrieval attempts and, accordingly, should only have a reduced influence on later thoughts (e.g., Wang et al., 2019). However, in many situations, preventing retrieval may also hinder subsequent recall of the unwanted memory by non-inhibitory processes such as associative interference (Verde, 2013; Racsmány, Conway, Keresztes, \& Krajcsi, A., 2012; Tomlinson, Huber, Rieth, \& Davelaar, 2009; Hertel \& Calcaterra, 2005).

775 Interference may particularly contribute to forgetting following thought substitution, which likely strengthens the association between the cue (e.g., TOMATO) and the alternate thought or memory that participants had retrieved (e.g., CLOWN) to prevent the unwanted target memory from coming to mind (e.g. VEST). In fact, some authors have suggested that thought substitution may be predominantly achieved through interference (Racsmány et al., 2012; Hertel \& McDaniel, 2010; Bergström et al., 2009; cf., Benoit \& Anderson, 2012; see Belli, 2011, for extensive discussion), similarly to retroactive interference in the $A-B, A-C$ paradigm (Briggs, 1954). (Though 
recent evidence suggests that inhibition also plays a role in this paradigm. Hulbert \& Anderson, 2020).

Behavioral evidence for inhibitory versus non-inhibitory accounts of SIF is provided by studies that employed an independent probe procedure to assess forgetting (Anderson \& Green, 2001; Bergström, de Fockert, \& Richardson-Klavehn, 2009). In these studies, the suppressed memories are also probed with a new cue that has a strong pre-experimental association with the memory (e.g., its category; DRESS - $V$ for VEST). This test thus probes the memory while circumventing its association with the original cue (e.g., TOMATO). As such, SIF on an independent probe test is unlikely to be caused by associative interference (cf. Racsmány et al., 2012). Instead, it is more likely caused by a weakened representation of the suppressed memory, consistent with an inhibitory account of memory control.

Of the included studies, very few used an independent probe test. It is thus difficult to gauge the evidence for inhibitory versus non-inhibitory forgetting. However, an exploratory analysis of the four studies that included such a test (comprising eight effect sizes across healthy and (sub)clinical samples) revealed a trend for a small effect only, SMCC $=0.18,95 \%$ CI $[-0.0356,0.402], p=.088$. However, all of these studies provided unspecified instructions or used a thought substitution procedure, and particularly the latter has only inconsistently been associated with inhibitory forgetting (Bergström et al, 2009; Benoit \& Anderson, 2012). The efficacy of thought substitution in inducing inhibitory forgetting may hinge on the exact choice of the substitute memories and their relatedness to the unwanted memories (Benoit \& Anderson, 2012; Hertel \& McDaniel, 2010; Norman et al., 2007). These exploratory results should encourage future meta-analytical treatments of SIF as measured by independent probes (https://osf.io/hmctu), with particular attention to the instructed mechanism. 


\subsection{Compromised suppression-induced forgetting in mental disorders associated with intrusive thoughts}

The meta-analysis of the non-clinical samples indicated that SIF is a replicable phenomenon in the general population. We had further hypothesized that it may constitute a beneficial coping mechanism to deal with unwanted thoughts and

815 memories. If this were the case, we would expect individuals who find it more difficult to contain intrusive thoughts in their everyday life to be worse at suppression. To test this account, we meta-analyzed groups of participants with mental disorders characterized by intrusive thoughts, or who were sub-clinical yet potentially susceptible to such issues as indicated by related trait measures.

820 In line with our hypothesis, this analysis did not provide evidence for a SIF effect across the clinical and sub-clinical samples, with the exception of repressive copers. Critically, SIF in the (sub)clinical groups was moreover significantly smaller among the group of emotional disorders (i.e., depression and anxiety) than in the healthy control samples.

The moderator analysis also provided no evidence for SIF in (sub)clinical participants following either direct retrieval suppression or thought substitution. These results may suggest that individuals with emotional disorders do not fundamentally profit from instructed mechanisms. However, only a few studies had employed thought substitution, and all of these examined individuals with depression. Thus, results concerning thought substitution should be interpreted carefully.

This is particularly the case given the observed variance across studies, suggesting that the effectiveness of this mechanism may hinge on specific features related to the experimental design. For example, in general, thought substitution may be more effective in inducing forgetting, when individuals are provided with substitutes that are unrelated to the unwanted memories (Hertel \& Calcaterra, 2005; Benoit \& Anderson, 2012) than when they first have to generate the substitutes themselves (Bergström et al., 2009). Retrieving a substitute (e.g., SHIRT) that is strongly related to the unwanted memory (e.g., VEST) may have the unintended consequence of 
leading to a co-activation and subsequent strengthening of the latter's representation 840 (see also Bäuml \& Hartinger, 2002; Goodmon \& Anderson, 2011; Anderson, Green, \& McCulloch, 2000). To aid depressed individuals with deficient cognitive control, it would thus be critical to carefully consider the nature of the provided substitutes and their relationship to the unwanted memories (Hertel \& Calcaterra, 2005).

We observed a large impairment in SIF for participants with PTSD, GAD, or elevated 845 anxiety. These data thus corroborate prior evidence from individual studies that had reported a negative association between SIF and trait anxiety (Benoit et al., 2016; Waldhauser et al., 2018), poor thought control ability (Catarino et al., 2015), depressed mood (Zhang et al., 2016), or rumination (Fawcett et al., 2015). Moreover, a similar pattern has been reported on indirect measures of memory performance 850 (Hertel et al. 2018) and implicit tests (Mary et al., 2020). The pattern is also consistent with several studies that similarly associated clinical phenomena with related deficient control processes at retrieval (e.g., GAD, Kircanski et al., 2016; clinical depression, Groome \& Sterkaj, 2008; substance-related and addictive disorders, Stramaccia, Penolazzi, Monego, Manzan, Castelli, \& Galfano, 2017; eating disorders, Stramaccia, Penolazzi, Libardi, Genovese, Castelli, Palomba, \& Galfano). More generally, these results are in line with a recent meta-analysis that associated broader cognitive control deficits with negative thinking (Zetsche, Bürkner, \& Schulze, 2018).

However, we found that repressive copers showed stronger SIF than the control 860 samples. This may be consistent with the observation that less anxious individuals are better at intentionally inducing forgetting, given that a repressive coping style has been associated with low trait anxiety (Kim et al., 2007; Myers, 2010). It may also be consistent with the observation that individuals who spontaneously engage in suppressive negative thoughts over the course of their lives display higher SIF 865 (Hulbert \& Anderson, 2018). However, results for this cluster were based on three studies only, and therefore especially require further corroboration. 
The present meta-analyses focused on memory control at the stage of retrieval. We consider this a relevant stage for the treatment of psychological disorders, seeing that patients with PTSD, for example, tend to seek help after intrusive memories would already have had time to consolidate (Marks et al., 2018). Due to the delay between initial experience and subsequent treatment, it may often not be feasible to administer potential interventions at earlier stages (see Visser et al., 2018), such as the recently proposed computer game-based treatment (Iyadurai et al., 2018b) or pharmacological interventions (e.g., Zohar, Sonnino, Juven-Wetzler, \& Cohen, 2009) 875 that are effective in the early hours prior to the consolidation of traumatic experiences. Similarly, psychological conditions such as depression and anxiety are also characterized by a problematic focus on past memories in the form of rumination (Koval, Kuppens, Allen, Sheeber, 2012; Michael, Halligan, Clark, \& Ehlers, 2007).

The observed relationship between suppression and mental well-being may constitute 880 a conundrum when also considering clinical evidence that ties suppression to negative outcomes in trauma-related disorders (Holmes, Moulds, \& Kavanagh, 2007). We certainly want to emphasize that we do not claim that it is always beneficial to try to suppress unwanted memories. In general, it is critical to engage with negative life experiences and emotions and to integrate them into who we are (see also Biglan, Hayes, \& Pistorello, 2008; for examples of negative consequences of suppression in different contexts, see Le \& Impett, 2016; Srivastava, Tamir, McGonigal, John, \& Gross, 2009; Dalgleish \& Yiend, 2006). However, we also think that there are several factors that can reconcile a beneficial take on suppression with the apparently contradicting clinical experience.

890 First, if the ability to suppress memories is deficient in people who experience intrusive memories, then asking them to suppress an unwanted memory may in fact have the paradoxical effect of aggravating symptoms. That is, for them, attempts to suppress may be bound to fail and thus counterproductively induce rehearsal - and thus strengthening - of unwanted memories. 
895 Secondly, there seems to be a difference between the notion of suppression as framed in the Think/No-Think literature versus the literature on cognitive-behavioral therapy and emotion regulation (Engen \& Anderson, 2018). Specifically, direct retrieval suppression should not be confused with expressive suppression. Expressive suppression refers to the act of voluntarily inhibiting overt expressions of one's emotional states - for instance, facial expressions or tone of voice (Suchy, 2015). This, in turn, has been extensively associated with poorer well-being (Haga, Kraft, \& Corby, 2009; Moore, Zoellner, \& Mollenholt, 2008).

Finally, as pointed out by Visser et al. (2018), it may well turn out to be critical what aspects of a memory are being targeted. In particular, these authors argue that it 905 may be beneficial to spare the voluntary access to some declarative components of a trauma memory (e.g., what happened) while attenuating the intrusiveness of unwanted fragments and their aversive emotional component.

These issues notwithstanding, we propose that memory suppression can serve as a mechanism that helps us control the intrusive retrieval of unwanted memories

910 (Anderson et al., 2004; Depue, et al., 2007; Benoit, Hulbert, Huddleston, \& Anderson, 2015).

\subsection{Caveats}

Importantly, due to the designs of the primary studies, we are not able to infer a 915 causal relationship between reduced SIF and psychopathology. Prospective studies are needed to disentangle whether SIF impairments precede or follow psychopathology, and to determine their potential role as a disorder-maintaining factor. In this respect, the objective difficulty of obtaining large clinical samples and adequate matched controls, combined with the relatively small effect size (at least 920 when studies do not prescribe a specific suppression mechanism), call for joint efforts to investigate such causal relationships.

We also want to point out some limitations of the extant literature and the current meta-analyses. Based on the meta-analytical effect sizes and the large uncertainty 
associated with them, we note that the primary studies had used fairly low sample sizes overall (on average, about 24 participants per group), and that they thus were certainly low-powered. Note, however, that studies prescribing either thought substitution or direct retrieval suppression yielded considerably greater effect sizes. Furthermore, the limited number of effects in each clinical cluster did not allow us to conduct more fine-grained analyses such as for a possible gradient of impairment associated with the severity of the disorders.

Our conclusions should thus be evaluated with respect to the low power of some of the primary data and the relatively high heterogeneity of some of the meta-analytic models. In addition, we had limited means to assess the impact of some of the chosen moderators. For this reason, in future meta-analytic endeavors, it would be worthwhile to examine other aspects of the Think/No-Think task that may constitute boundary conditions to the efficacy of SIF (such as the number of suppressed target memories, specific features of the learning procedure such as the encoding task, the relatedness of cues and targets, and the maximum allotted time for memory retrieval on the final test). A further synthesis of the published literature can also confirm the magnitude of SIF in the broader population (rather than in healthy versus (sub)clinical populations) (e.g., https://osf.io/hmctu).

Finally, methods such as Egger's regression may underestimate the presence of publication bias, especially so in the context of highly heterogeneous models (Renkewitz \& Keiner, preprint). (See also Appendix 2 for the surprising observation 945 that the inclusion of unpublished studies that yielded null results or even reversed effects can lead to a stronger impression of publication bias as estimated by the trimand-fill procedure.) Therefore, any meta-analytical efforts should ideally be complemented by pre-registered, large-scale replication attempts. Nonetheless, we suggest that meta-analyses currently provide the best available evidence on SIF and its disturbance in clinical disorders. 


\subsection{Conclusions}

In light of the present results, we suggest that SIF is the replicable hallmark of a process that allows us to voluntarily prevent memory retrieval. Importantly, the observation that this ability is associated with psychological well-being indicates that it may indeed constitute an adaptive coping mechanism. We certainly neither propose that preventing retrieval is always beneficial nor do we suggest that fostering suppression would necessarily be an adequate therapeutic intervention. Yet, in our everyday life, it may help us control intrusive and unwanted thoughts and thus allow us to edit the contents of our memories.

\section{Role of funding sources}

RGB, DFS, KMR, and A-KM were funded by a Max Planck Research Group awarded to RGB. JMF was funded by an NSERC Discovery Grant.

\section{Acknowledgements}

We thank Kerstin Flake and Heike Schmidt Duderstedt for their assistance in creating the figures.

\section{Context}

Our lab examines intentional forgetting as an adaptive memory process and has contributed to the understanding of the underlying neural mechanisms (e.g., Benoit \& Anderson, 2012; Benoit et al., 2015). Going forward, we think it is important to better characterize the contribution of this process to maintaining mental well-being. As such, we thought it prudent to analyze the literature for an estimate of the effect size of SIF in healthy and (sub)clinical populations. 


\section{References}

(Articles included in the analyses are marked with $a *$.)

980

Akaike H. (1998) Information Theory and an Extension of the Maximum Likelihood Principle. In: Parzen E., Tanabe K., Kitagawa G. (eds) Selected Papers of Hirotugu Akaike. Springer Series in Statistics (Perspectives in Statistics). Springer, New York, NY. https://doi.org/10.1007/978-1-4612-1694-0_15

Algarabel, S., Luciano, J. V., \& Martínez, J. L. (2006). Inhibitory Voluntary Control of Memory: Effect of Stimulus Onset Asynchrony on Reaction Time to Suppressed Memories. Psicologica: International Journal of Methodology and Experimental Psychology, 27(1), 57-77.

American Psychiatric Association. (2013). Diagnostic and statistical manual of mental disorders (5th ed.). Washington, DC: Author.

Anderson, M. C., \& Green, C. (2001). Suppressing unwanted memories by executive control. Nature, 410(6826), 366.

Anderson, M. C., Green, C., \& McCulloch, K. C. (2000). Similarity and inhibition in long-term memory: evidence for a two-factor theory. Journal of Experimental Psychology: Learning, Memory, and Cognition, 26(5), 1141.

Anderson, M. C., Ochsner, K. N., Kuhl, B., Cooper, J., Robertson, E., Gabrieli, S. W., ... \& Gabrieli, J. D. (2004). Neural systems underlying the suppression of unwanted memories. Science, 303(5655), 232-235.

Anderson, M. C., \& Huddleston, E. (2012). Towards a cognitive and neurobiological 1000 model of motivated forgetting. In True and false recovered memories (pp. 53120). Springer, New York, NY.

Anderson, M. C., \& Hanslmayr, S. (2014). Neural mechanisms of motivated forgetting. Trends in Cognitive Sciences, 18(6), 279-292.

Belli, R. F. (Ed.). (2011). True and false recovered memories: Toward a reconciliation of the debate (Vol. 58). Springer Science \& Business Media. 
Bäuml, K. H., \& Hartinger, A. (2002). On the role of item similarity in retrievalinduced forgetting. Memory, 10(3), 215-224.

Benoit, R. G., \& Anderson, M. C. (2012). Opposing mechanisms support the voluntary forgetting of unwanted memories. Neuron, 76(2), 450-460.

1010 Benoit, R. G., Hulbert, J. C., Huddleston, E., \& Anderson, M. C. (2015). Adaptive topdown suppression of hippocampal activity and the purging of intrusive memories from consciousness. Journal of Cognitive Neuroscience, 27(1), 96111.

Benoit, R. G., Davies, D. J., \& Anderson, M. C. (2016). Reducing future fears by 1015 suppressing the brain mechanisms underlying episodic simulation. Proceedings of the National Academy of Sciences, 113(52), E8492-E8501.

Bergström, Z. M., Velmans, M., de Fockert, J., \& Richardson-Klavehn, A. (2007). ERP evidence for successful voluntary avoidance of conscious recollection. Brain research, 1151, 119-133.

1020 Bergström, Z. M., de Fockert, J. W., \& Richardson-Klavehn, A. (2009). ERP and behavioural evidence for direct suppression of unwanted memories. NeuroImage, 48(4), 726-737.

Bernstein, E. M., \& Putnam, F. W. (1986). Development, reliability, and validity of a dissociation scale. Journal of nervous and mental disease.

1025 Bjork, R. A. (1970). Positive forgetting: The noninterference of items intentionally forgotten. Journal of Verbal Learning and Verbal Behavior, 9(3), 255-268.

Bjork, R. A. (1989). Retrieval inhibition as an adaptive mechanism in human memory. In H. L. Roediger III \& F. I. M. Craik (Eds.), Varieties of memory and consciousness: Essays in honour of Endel Tulving (p. 309-330). Lawrence Erlbaum Associates, Inc.

Bjork, E. L., \& Bjork, R. A. (1996). Continuing influences of to-be-forgotten information. Consciousness and cognition, 5(1-2), 176-196. 
Brewin, C. R. (2014). Episodic memory, perceptual memory, and their interaction: foundations for a theory of posttraumatic stress disorder. Psychological 1035 Bulletin, 140(1), 69.

Briggs, G. E. (1954). Acquisition, extinction, and recovery functions in retroactive inhibition. Journal of Experimental Psychology, 47(5), 285-293. https://doi.org/10.1037/h0060251

Bulevich, J. B., Roediger, H. L., Balota, D. A., \& Butler, A. C. (2006). Failures to find 1040 suppression of episodic memories in the think/no-think paradigm. Memory \& cognition, 34(8), 1569-1577.

Card, N. A. (2015). Applied meta-analysis for social science research. Guilford Publications.

*Catarino, A., Küpper, C. S., Werner-Seidler, A., Dalgleish, T., \& Anderson, M. C. 1045 (2015). Failing to forget: Inhibitory-control deficits compromise memory suppression in posttraumatic stress disorder. Psychological Science, 26(5), 604-616.

Cochran, W. G. (1954). Thce combination of estimates from different experiments. Biometrics, 10(1), 101-129.

1050 Cook, R. D., \& Weisberg, S. (1982). Residuals and influence in regression. New York: Chapman and Hall.

Cooper, H., Hedges, L. V., \& Valentine, J. C. (Eds.). (2019). The handbook of research synthesis and meta-analysis. Russell Sage Foundation.

Dalgleish, T., \& Yiend, J. (2006). The effects of suppressing a negative 1055 autobiographical memory on concurrent intrusions and subsequent autobiographical recall in dysphoria. Journal of Abnormal Psychology, 115(3), 467.

Depue, B. E., Curran, T., \& Banich, M. T. (2007). Prefrontal regions orchestrate suppression of emotional memories via a two-phase process. Science, $317(5835), 215-219$. 
*Depue, B. E., Burgess, G. C., Willcutt, E. G., Ruzic, L., \& Banich, M. T. (2010). Inhibitory control of memory retrieval and motor processing associated with the right lateral prefrontal cortex: evidence from deficits in individuals with ADHD. Neuropsychologia, 48(13), 3909-3917.

1065 Detre, G. J., Natarajan, A., Gershman, S. J., \& Norman, K. A. (2013). Moderate levels of activation lead to forgetting in the think/no-think paradigm. Neuropsychologia, 51(12), 2371-2388.

*Dieler, A. C., Herrmann, M. J., \& Fallgatter, A. J. (2014). Voluntary suppression of thoughts is influenced by anxious and ruminative tendencies in healthy volunteers. Memory, 22(3), 184-193.

*Diwadkar, V. A., Re, M., Cecchetto, F., Garzitto, M., Piccin, S., Bonivento, C., ... \& Brambilla, P. (2017). Attempts at memory control induce dysfunctional brain activation profiles in Generalized Anxiety Disorder: An exploratory fMRI study. Psychiatry Research: Neuroimaging, 266, 42-52.

1075 Drevon, D., Fursa, S. R., \& Malcolm, A. L. (2017). Intercoder reliability and validity of WebPlotDigitizer in extracting graphed data. Behavior Modification, 41(2), 323-339.

Duval, S., \& Tweedie, R. (2000). Trim and fill: a simple funnel- plot-based method of testing and adjusting for publication bias in meta- analysis. Biometrics, $56(2), 455-463$.

Egger, M., Smith, G. D., Schneider, M., \& Minder, C. (1997). Bias in meta-analysis detected by a simple, graphical test. British Medical Journal, 315, 629-634.

Ehlers, A., Hackmann, A., \& Michael, T. (2004). Intrusive re- experiencing in posttraumatic stress disorder: Phenomenology, theory, and therapy. Memory, $12(4), 403-415$.

Engen, H. G., \& Anderson, M. C. (2018). Memory control: a fundamental mechanism of emotion regulation. Trends in Cognitive Sciences.

Eysenck, S. B., Eysenck, H. J., \& Barrett, P. (1985). A revised version of the psychoticism scale. Personality and individual differences, 6(1), 21-29. 
*Fawcett, J. M., Benoit, R. G., Gagnepain, P., Salman, A., Bartholdy, S., Bradley, C., ... \& Anderson, M. C. (2015). The origins of repetitive thought in rumination: Separating cognitive style from deficits in inhibitory control over memory. Journal of Behavior Therapy and Experimental Psychiatry, 47, 1-8.

Fawcett, J. M., \& Hulbert, J. C. (2020). The Many Faces of Forgetting: Toward a Constructive View of Forgetting in Everyday Life. Journal of Applied Research in Memory and Cognition, 9(1), 1-18.

Gaddy, M. A., \& Ingram, R. E. (2014). A meta-analytic review of mood-congruent implicit memory in depressed mood. Clinical Psychology Review, 34(5), 402416.

1100 Gagnepain, P., Henson, R. N., \& Anderson, M. C. (2014). Suppressing unwanted memories reduces their unconscious influence via targeted cortical inhibition. Proceedings of the National Academy of Sciences, 201311468.

Goodmon, L. B., \& Anderson, M. C. (2011). Semantic integration as a boundary condition on inhibitory processes in episodic retrieval. Journal of Experimental Psychology: Learning, Memory, and Cognition, 37(2), 416.

Goodwin, G. M. (2015). The overlap between anxiety, depression, and obsessivecompulsive disorder. Dialogues in Clinical Neuroscience, 17(3), 249.

Goschke, T. (2014). Dysfunctions of decision- making and cognitive control as transdiagnostic mechanisms of mental disorders: advances, gaps, and needs in current research. International Journal of Methods in Psychiatric Research, 23(S1), 41-57.

Groome, D., \& Sterkaj, F. (2010). Retrieval-induced forgetting and clinical depression. Cognition and Emotion, 24(1), 63-70.

Hackmann, A., Ehlers, A., Speckens, A., \& Clark, D. M. (2004). Characteristics and content of intrusive memories in PTSD and their changes with treatment. Journal of Traumatic Stress: Official Publication of The International Society for Traumatic Stress Studies, 17(3), 231-240. 
Haga, S. M., Kraft, P., \& Corby, E. K. (2009). Emotion regulation: Antecedents and well-being outcomes of cognitive reappraisal and expressive suppression in cross-cultural samples. Journal of Happiness Studies, 10(3), 271-291.

Biglan, A., Hayes, S. C., \& Pistorello, J. (2008). Acceptance and commitment: Implications for prevention science. Prevention Science, 9(3), 139-152.

Hair, J. F., Black, W. C., Babin, B. J., Anderson, R. E., \& Tatham, R. L. (1998). Multivariate data analysis (Vol. 5, No. 3, pp. 207-219). Upper Saddle River, NJ: Prentice hall.

Hedges, L. V., \& Olkin, I. (1985). Statistical methods for meta-analysis. San Diego, CA: Academic Press.

Hedges, L. V., Tipton, E., \& Johnson, M. C. (2010). Robust variance estimation in meta- regression with dependent effect size estimates. Research synthesis methods, 1(1), 39-65.

Hertel, P. T. (1997). On the contributions of deficient cognitive control to memory impairments in depression. Cognition \& Emotion, 11(5-6), 569-583.

Hertel, P. T. (1998). Relation between rumination and impaired memory in dysphoric moods. Journal of Abnormal Psychology, 107(1), 166.

1135 *Hertel, P. T., \& Gerstle, M. (2003). Depressive deficits in forgetting. Psychological Science, 14(6), 573-578.

Hertel, P. T., \& Calcaterra, G. (2005). Intentional forgetting benefits from thought substitution. Psychonomic Bulletin \& Review, 12(3), 484-489.

Hertel, P. T. (2007). Impairments in inhibition or cognitive control in psychological disorders. Applied and Preventive Psychology, 12(3), 149-153.

*Hertel, P. T., \& Mahan, A. (2008). Depression-related differences in learning and forgetting responses to unrelated cues. Acta Psychologica, 127(3), 636-644.

*Hertel, P., \& McDaniel, L. (2010). The suppressive power of positive thinking: Aiding suppression-induced forgetting in repressive coping. Cognition and Emotion, 24(7), 1239-1249. 
Hertel, P. T., Large, D., Stück, E. D., \& Levy, A. (2012). Suppression-induced forgetting on a free-association test. Memory, 20(2), 100-109.

Hertel, P. T., Maydon, A., Ogilvie, A., \& Mor, N. (2018). Ruminators (Unlike Others) Fail to Show Suppression-Induced Forgetting on Indirect Measures of Memory. Clinical Psychological Science, 6(6), 872-881.

Hertwig, R., \& Engel, C. (2016). Homo ignorans: Deliberately choosing not to know. Perspectives on Psychological Science, 11(3), 359-372.

Higgins, J. P. T., \& Green, S. (Eds.). (2011). Cochrane handbook for systematic reviews of interventions. John Wiley \& Sons.

1155 Higgins, J. P., Thompson, S. G., Deeks, J. J., \& Altman, D. G. (2003). Measuring inconsistency in meta-analyses. BMJ: British Medical Journal, 327(7414), 557.

Holmes, E. A., Moulds, M. L., \& Kavanagh, D. (2007). Memory suppression in PTSD treatment?. Science, 318(5857), 1722-1722.

Hulbert, J. C., \& Anderson, M. C. (2020). Does retrieving a memory insulate it against memory inhibition? A retroactive interference study. Memory, 1-16.

Hulbert, J. C., \& Anderson, M. C. (2018). What doesn't kill you makes you stronger: Psychological trauma and its relationship to enhanced memory control. Journal of Experimental Psychology: General, 147(12), 1931.

Hulbert, J. C., Hirschstein, Z., Brontë, C. A., \& Broughton, E. (2018). Unintended side effects of a spotless mind: theory and practice. Memory, 26(3), 306-320.

Iyadurai, L., Visser, R. M., Lau-Zhu, A., Porcheret, K., Horsch, A., Holmes, E. A., \& James, E. L. (2018a). Intrusive memories of trauma: $A$ target for research bridging cognitive science and its clinical application. Clinical Psychology Review, 69, 67-82.

1170 Iyadurai, L., Blackwell, S. E., Meiser-Stedman, R., Watson, P. C., Bonsall, M. B., Geddes, J. R., ... \& Holmes, E. A. (2018b). Preventing intrusive memories after trauma via a brief intervention involving Tetris computer game play in the emergency department: a proof-of-concept randomized controlled trial. Molecular psychiatry, 23(3), 674. 
1175 IntHout, J., Ioannidis, J. P., Rovers, M. M., \& Goeman, J. J. (2016). Plea for routinely presenting prediction intervals in meta-analysis. BMJ open, 6(7), e010247.

*Joormann, J., Hertel, P. T., Brozovich, F., \& Gotlib, I. H. (2005). Remembering the good, forgetting the bad: intentional forgetting of emotional material in depression. Journal of Abnormal Psychology, 114(4), 640.

1180 *Joormann, J., Hertel, P. T., LeMoult, J., \& Gotlib, I. H. (2009). Training forgetting of negative material in depression. Journal of Abnormal Psychology, 118(1), 34.

*Kim, K., Yi, D., Yang, E., \& Lee, K. (2007). What makes repressors good suppressors? The effect of trait anxiety. Korean Journal of Psychology, 26, 261-277.

1185 *Kim, D. Y., Oh, D. H., Kim, S. H., Sim, K. B., \& Lee, J. H. (2013). Effects of intentional suppression of recall of unwanted images in repressors and nonrepressors. Social Behavior and Personality: an international journal, $41(2), 319-326$.

Kircanski, K., Joormann, J., \& Gotlib, I. H. (2012). Cognitive aspects of depression. Wiley Interdisciplinary Reviews: Cognitive Science, 3(3), 301-313.

*Kircanski, K., Johnson, D. C., Mateen, M., Bjork, R. A., \& Gotlib, I. H. (2016). Impaired retrieval inhibition of threat material in generalized anxiety disorder. Clinical Psychological Science, 4(2), 320-327.

Konstantopoulos, S. (2011). Fixed effects and variance components estimation in three- level meta- analysis. Research Synthesis Methods, 2(1), 61-76.

Koval, P., Kuppens, P., Allen, N. B., \& Sheeber, L. (2012). Getting stuck in depression: The roles of rumination and emotional inertia. Cognition \& emotion, 26(8), 1412-1427.

*Küpper, C. S., Benoit, R. G., Dalgleish, T., \& Anderson, M. C. (2014). Direct 1200 suppression as a mechanism for controlling unpleasant memories in daily life. Journal of Experimental Psychology: General, 143(4), 1443. 
Le, B. M., \& Impett, E. A. (2016). The costs of suppressing negative emotions and amplifying positive emotions during parental caregiving. Personality and Social Psychology Bulletin, 42(3), 323-336.

1205 Lipsey, M. W., \& Wilson, D. B. (2001). Practical meta-analysis. Sage Publications, Inc.

Luciano, J. V., Algarabel, S., Tomás, J. M., \& Martínez, J. L. (2005). Development and validation of the thought control ability questionnaire. Personality and Individual Differences, 38(5), 997-1008.

1210 Marks, E. H., Franklin, A. R., \& Zoellner, L. A. (2018). Can't get it out of my mind: A systematic review of predictors of intrusive memories of distressing events. Psychological Bulletin, 144(6), 584.

*Marzi, T., Regina, A., \& Righi, S. (2014). Emotions shape memory suppression in trait anxiety. Frontiers in Psychology, 4, 1001.

1215 Mary, A., Dayan, J., Leone, G., Postel, C., Fraisse, F., Malle, C., ... \& Gagnepain, P. (2020). Resilience after trauma: The role of memory suppression. Science, 367(6479).

Matt, G. E., Vázquez, C., \& Campbell, W. K. (1992). Mood-congruent recall of affectively toned stimuli: A meta-analytic review. Clinical Psychology Review, $12(2), 227-255$.

McGeoch, J. A. (1932). Forgetting and the law of disuse. Psychological review, 39(4), 352.

McGraw, K. O., \& Wong, S. P. (1996). Forming inferences about some intraclass correlation coefficients. Psychological methods, 1(1), 30.

1225 McTeague, L. M., Goodkind, M. S., \& Etkin, A. (2016). Transdiagnostic impairment of cognitive control in mental illness. Journal of Psychiatric Research, 83, 37-46.

Mecklinger, A., Parra, M., \& Waldhauser, G. T. (2009). ERP correlates of intentional forgetting. Brain Research, 1255, 132-147.

Michael, T., Halligan, S. L., Clark, D. M., \& Ehlers, A. (2007). Rumination in posttraumatic stress disorder. Depression and anxiety, 24(5), 307-317. 
Moher, D., Liberati, A., Tetzlaff, J., \& Altman, D. G. (2009). Preferred reporting items for systematic reviews and meta-analyses: the PRISMA statement. Annals of internal medicine, 151(4), 264-269.

Moore, S. A., Zoellner, L. A., \& Mollenholt, N. (2008). Are expressive suppression and cognitive reappraisal associated with stress-related symptoms? Behaviour research and therapy, 46(9), 993-1000.

Nakagawa, S., \& Santos, E. S. (2012). Methodological issues and advances in biological meta-analysis. Evolutionary Ecology, 26(5), 1253-1274.

*Nemeth, V. L., Kurgyis, E., Csifcsak, G., Maraz, A., Almasi, D. A., Drotos, G., ... \& Must, A. (2014). The impact of intermediate-term alcohol abstinence on memory retrieval and suppression. Frontiers in Psychology, 5, 1396.

Nørby, S. (2015). Why forget? On the adaptive value of memory loss. Perspectives on Psychological Science, 10(5), 551-578.

Nørby, S. (2018). Forgetting and emotion regulation in mental health, anxiety and depression. Memory, 26(3), 342-363.

*Noreen, S., \& Ridout, N. (2016a). Intentional forgetting in dysphoria: investigating the inhibitory effects of thought substitution using independent cues. Journal of Behavior Therapy and Experimental Psychiatry, 52, 110-118.

*Noreen, S., \& Ridout, N. (2016b). Examining the impact of thought substitution on intentional forgetting in induced and naturally occurring dysphoria. Psychiatry Research, 241, 280-288.

*Noreen, S., Cooke, R. \& Ridout, N. Investigating the mediating effect of working memory on intentional forgetting in dysphoria. Psychological Research (2019). https://doi.org/10.1007/s00426-019-01225-y

1255 Norman, K. A., Newman, E. L., \& Detre, G. (2007). A neural network model of retrieval-induced forgetting. Psychological review, 114(4), 887.

Payne, L., \& Sekuler, R. (2014). The importance of ignoring: Alpha oscillations protect selectivity. Current directions in psychological science, 23(3), 171-177. 
Peters, J. L., Sutton, A. J., Jones, D. R., Abrams, K. R., \& Rushton, L. (2006). Comparison of two methods to detect publication bias in meta-analysis. Jama, 295(6), 676-680.

Peters, J. L., Sutton, A. J., Jones, D. R., Abrams, K. R., \& Rushton, L. (2008). Contour-enhanced meta-analysis funnel plots help distinguish publication bias from other causes of asymmetry. Journal of Clinical Epidemiology, 61(10), 991-996.

R Development Core Team (2008). R: A Language and Environment for Statistical Computing. R Foundation for Statistical Computing, Vienna, Austria. http://www.r-project.org/

Racsmány, M., Conway, M. A., Keresztes, A., \& Krajcsi, A. (2012). Inhibition and interference in the think/no-think task. Memory \& Cognition, 40(2), 168-176.

Renkewitz, F., \& Keiner, M. (2019). How to detect publication bias in psychological research: A comparative evaluation of six statistical methods. Zeitschrift für Psychologie, 227(4), 261-279. http://dx.doi.org/10.1027/21512604/a000386

1275 Rohatgi, A. (2017). WebPlotDigitizer, Version 3.11 [Computer software]. http://arohatgi.info/WebPlotDigitizer

*Ryckman, N. (2015). Exploring intentional forgetting: Using novel think/no-think paradigms to investigate memory suppression (Doctoral dissertation, ResearchSpace@ Auckland).

*Sacchet, M. D., Levy, B. J., Hamilton, J. P., Maksimovskiy, A., Hertel, P. T., Joormann, J., ... \& Gotlib, I. H. (2017). Cognitive and neural consequences of memory suppression in major depressive disorder. Cognitive, Affective, \& Behavioral Neuroscience, 17(1), 77-93.

Sahakyan, L., Waldum, E. R., Benjamin, A. S., \& Bickett, S. P. (2009). Where is the 1285 forgetting with list-method directed forgetting in recognition?. Memory \& cognition, 37(4), 464-476. 
*Salamé, P., \& Danion, J. M. (2007). Inhibition of inappropriate responses is preserved in the think-no-think and impaired in the random number generation tasks in schizophrenia. Journal of the International Neuropsychological Society, 13(2), 277-287.

van Schie, K., \& Anderson, M. C. (2017). Successfully controlling intrusive memories is harder when control must be sustained. Memory, 25(9), 1201-1216.

Spiess, A-N. (2018). qpcR: Modelling and analysis of real-time PCR data. R package version 1.4 -1. https://cran.r-project.org/web/packages/qpcR/index.html

1295 Srivastava, S., Tamir, M., McGonigal, K. M., John, O. P., \& Gross, J. J. (2009). The social costs of emotional suppression: A prospective study of the transition to college. Journal of personality and social psychology, 96(4), 883.

*Stephens, E., Braid, A., \& Hertel, P. T. (2013). Suppression-induced reduction in the specificity of autobiographical memories. Clinical Psychological Science, $1(2), 163-169$.

Sterne, J. A. C., \& Egger, M. (2005). Regression methods to detect publication and other bias in meta-analysis. In H. Rothstein, A. Sutton, \& M. Borenstein (Eds.), Publication bias in meta-analysis: Prevention, assessment and adjustments (pp. 99 -110). Chichester, England: Wiley.

Stramaccia, D. F., Penolazzi, B., Libardi, A., Genovese, A., Castelli, L., Palomba, D., \& Galfano, G. (2018). Control over interfering memories in eating disorders. Journal of clinical and experimental neuropsychology, 40(1), 30-44.

Stramaccia, D. F., Penolazzi, B., Monego, A. L., Manzan, A., Castelli, L., \& Galfano, G. (2017). Suppression of competing memories in substance-related and addictive disorders: A retrieval-induced forgetting study. Clinical Psychological Science, 5(2), 410-417.

Suchy, Y. (2015). Executive functioning: A comprehensive guide for clinical practice. Oxford University Press. 
Sullivan, D. R., Marx, B., Chen, M. S., Depue, B. E., Hayes, S. M., \& Hayes, J. P. (2019). Behavioral and neural correlates of memory suppression in PTSD. Journal of psychiatric research.

Thorndike, E. L. (1913). The psychology of learning. Teachers College, Columbia University.

Thornton, A., \& Lee, P. (2000). Publication bias in meta-analysis: its causes and consequences. Journal of Clinical Epidemiology, 53(2), 207-216.

Tomlinson, T. D., Huber, D. E., Rieth, C. A., \& Davelaar, E. J. (2009). An interference account of cue-independent forgetting in the no-think paradigm. Proceedings of the National Academy of Sciences, 106(37), 15588-15593.

Tulving, E. (1974). Cue-dependent forgetting: When we forget something we once knew, it does not necessarily mean that the memory trace has been lost; it may only be inaccessible. American Scientist, 62(1), 74-82.

Underwood, B. J. (1957). Interference and forgetting. Psychological review, 64(1), 49.

Verde, M. F. (2013). Retrieval-induced forgetting in recall: Competitor interference revisited. Journal of Experimental Psychology: Learning, Memory, and Cognition, 39(5), 1433.

Viechtbauer, W. (2010). Conducting meta-analyses in R with the metafor package. Journal of Statistical Software, 36(3).

Viechtbauer, W., \& Cheung, M. W. L. (2010). Outlier and influence diagnostics for meta- analysis. Research synthesis methods, 1(2), 112-125.

Visser, R. M., Lau-Zhu, A., Henson, R. N., \& Holmes, E. A. (2018). Multiple memory systems, multiple time points: how science can inform treatment to control the expression of unwanted emotional memories. Phil. Trans. R. Soc. B, 373(1742), 20170209.

1340 Wagenmakers, E. J., \& Farrell, S. (2004). AIC model selection using Akaike weights. Psychonomic bulletin \& review, 11(1), 192-196. 
*Waldhauser, G. T., Dahl, M. J., Ruf-Leuschner, M., Müller-Bamouh, V., Schauer, M., Axmacher, N., ... \& Hanslmayr, S. (2018). The neural dynamics of deficient memory control in heavily traumatized refugees. Scientific Reports, 8(1), 13132.

Wang, Y., Luppi, A., Fawcett, J., \& Anderson, M. C. (2019). Reconsidering unconscious persistence: Suppressing unwanted memories reduces their indirect expression in later thoughts. Cognition, 187, 78-94.

Wessel, I., Albers, C. J., Zandstra, A. E., \& Heininga, V. E. (2020, March 27). A Multiverse Analysis of Early Attempts to Replicate Memory Suppression with the Think/No-think Task. https://doi.org/10.31234/osf.io/2dr9e

*Wessel, I., Wetzels, S., Jelicic, M., \& Merckelbach, H. (2005). Dissociation and memory suppression: A comparison of high and low dissociative individuals' performance on the think-no think task. Personality and Individual Differences, 39(8), 1461-1470.

Williams, A. D., Moulds, M. L., Grisham, J. R., Gay, P., Lang, T., Kandris, E., ... \& Yap, C. (2010). A psychometric evaluation of the Thought Control Ability Questionnaire (TCAQ) and the prediction of cognitive control. Journal of Psychopathology and Behavioral Assessment, 32(3), 397-405.

1360 World Health Organization. (2018). International statistical classification of diseases and related health problems (11th Revision). Retrieved from https://icd.who.int/browse11/l-m/en

Zetsche, U., Bürkner, P. C., \& Schulze, L. (2018). Shedding light on the association between repetitive negative thinking and deficits in cognitive control - a metaanalysis. Clinical Psychology Review.

*Zhang, D., Xie, H., Liu, Y., \& Luo, Y. (2016). Neural correlates underlying impaired memory facilitation and suppression of negative material in depression. Scientific Reports, 6, 37556.

Zohar, J., Sonnino, R., Juven-Wetzler, A., \& Cohen, H. (2009). Can posttraumatic 1370 stress disorder be prevented. CNS Spectr, 14(1 Suppl 1), 44-51. 


\section{Appendix 1 - simple random-effects models of only independent effect sizes}

Many of the included studies provided multiple estimates of SIF (i.e., one for each level of within-subject manipulations such as the number of repetitions). The main

1375 analysis is based on a multi-level random-effects model that comprehensively includes all of these estimates.

Here, we provide the results of complementary simpler random-effects models that include only one independent effect size from each independent group in each study. In particular, in case of multiple dependent effect sizes, we selected the one that 1380 constituted the standard manipulation (e.g., greater number of suppression repetitions) and/or outcome measure (binary rather than continuous measures of recall accuracy) in respect to the extant literature on the Think/No-Think task (as in a prior preprint on PsyArXiv doi: 10.31234/osf.io/5wynm) (see Card, 2012, pp. 192193; Cooper, Hedges, \& Valentine; 2019, p. 282; Higgins and Green, 2011, Chapter

1385 3; Lipsey and Wilson, 2001, p. 125). This approach presumably provides an estimate of the strongest manipulations (e.g., a greater rather than a lesser number of suppression repetitions). We perform these models separately for the healthy samples and the (sub)clinical samples. This approach also allows us to implement the trim-and-fill procedure (Duval \& Tweedie, 2000), in a further attempt to gauge the

1390 degree of publication bias. This procedure estimates the number of missing studies in the meta-analytic model due to publication bias and the impact that they might have on the meta-analytic effect size.

\section{Suppression-induced forgetting in healthy samples}

For the healthy samples (32 effects), there was a significant SIF effect of $0.27,95 \%$ 1395 CI $[0.16,0.39], 95 \%$ PI $[-.22, .77], p<.001$, with a large, significant heterogeneity, $I^{2}=58.05 \%, Q_{(31)}=76.83, p<.001$. Following trim and fill, with the insertion of data points for an estimated seven missing studies, the model remained significant with a reduced estimate of $0.17,95 \%$ CI $[0.05,0.30], 95 \%$ PI $[-.44, .79], p<.001$, 
and a large heterogeneity, $I^{2}=66.63 \%$ which was also significant, $Q_{(38)}=107.29, p$ $1400<.001$.

\section{No suppression-induced forgetting in (sub)clinical samples}

For the (sub)clinical samples (32 effects), there was a non-significant SIF effect of $0.08,95 \%$ CI $[-0.08,0.23], 95 \%$ PI $[-.68, .84], p=.320$, with a large, significant heterogeneity, $I^{2}=76.16 \%, Q_{(31)}=118.27, p<.001$. After trim and fill (with the 1405 insertion of data points for an estimated one missing study), the model remained non-significant with a reduced estimate of $0.06,95 \%$ CI $[-0.10,0.21], 95 \%$ PI $[-.73$, $.84], p<.001$, and a large, significant heterogeneity, $I^{2}=77.40 \%, Q_{(38)}=126.53$, $p<.001$. 


\section{Appendix 2 - simple random-effects models of average effect} sizes

To perform a trim-and-fill adjustment across all reported effect sizes (rather than the most standard measures of SIF), we first averaged all inter-dependent effect sizes of each study (i.e., the within-subject levels of a given independent variable). We then fitted separate simple random-effects models based on these average effect sizes.

1415 Suppression-induced forgetting in healthy samples: stronger correction

For the healthy samples (32 effects), there was a significant SIF effect of $0.26,95 \%$ CI $[0.14,0.39], 95 \%$ PI $[-.30, .82], p<.001$, and large heterogeneity, $I^{2}=64.05 \%$ which was also significant, $Q_{(31)}=85.22, p<.001$.

The trim-and-fill correction of the data from all studies added data points for an estimated eight missing studies, which resulted in a trend only for an effect of 0.13 , $95 \%$ CI $[-0.02,0.27], 95 \%$ PI $[-.65, .90], p=.084$, with a large heterogeneity, $I^{2}=$ $75.70 \%$ which was also significant, $Q_{(39)}=133.96, p<.001$.

We further sought to gauge the actual publication bias by refitting the trim-and-fill model based only on the published literature. This analysis thus excludes the three experiments (experiment 2, plus the combined experiments 3 and 4) by Ryckman (2015) that either yielded a null effect or, in fact, reversed SIF. Somewhat surprisingly, the trim-and-fill procedure exerted a lesser penalty on the estimate for only the published studies - with the insertion of six additional data points. Indeed, SIF remained significant with an effect of $0.18,95 \%$ CI $[0.03,0,32], 95 \%$ PI $[-0.55$, $0.90], p=.016$, with a large and significant heterogeneity, $Q_{(35)}=107.03, p<.001$. 


\section{No suppression-induced forgetting in (sub)clinical samples}

For the clinical samples (32 effects), SIF was not significant with an effect of 0.03 (95\% CI $[-0.11,0.17], 95 \%$ PI $[-.64, .69], p=.719$, and large, significant

1435 heterogeneity, $I^{2}=71.48 \%, Q_{(31)}=85.22, p<.001$.

The trim-and-fill correction of the averaged effect sizes did not yield any differences for the clinical samples.

In keeping with the same reasoning as for the healthy samples, we then removed the unpublished studies by Ryckman (2005) and re-estimated the trim-and-filled 1440 model. The procedure did no add any data points to the funnel plot and SIF was also not significant with an effect of $0.03,95 \%$ CI $[-0.12,0.18], 95 \%$ PI $[-.67, .74], p=$ .688 , and large, significant heterogeneity, $I^{2}=71.52 \%, Q_{(29)}=99.52, p<.001$. 